\title{
Understanding the physics of oligonucleotide microarrays: the Affymetrix spike-in data reanalysed
}

\author{
Conrad J Burden \\ Centre for Bioinformation Science, John Curtin School of Medical Research and Mathematical Sciences \\ Institute, Australian National University, Canberra, ACT 0200, Australia \\ E-mail: Conrad.Burden@anu.edu.au
}

Received 5 December 2007

Accepted for publication 6 March 2008

Published 26 March 2008

Online at stacks.iop.org/PhysBio/5/016004

\begin{abstract}
The Affymetrix U95 and U133 Latin-Square spike-in datasets are reanalysed, together with a dataset from a version of the U95 spike-in experiment without a complex non-specific background. The approach uses a physico-chemical model which includes the effects of the specific and non-specific hybridization and probe folding at the microarray surface, target folding and hybridization in the bulk RNA target solution and duplex dissociation during the post-hybridization washing phase. The model predicts a three-parameter hyperbolic response function that fits well with fluorescence intensity data from all the three datasets. The importance of the various hybridization and washing effects in determining each of the three parameters is examined, and some guidance is given as to how a practical algorithm for determining specific target concentrations might be developed.
\end{abstract}

\section{Notation}

$a:$

Physical background intensity measurement from factors such as reflection off the microarray surface and photomultiplier dark current. Assumed to be constant for all features on a microarray.

A: $\quad$ One of the three parameters in the hyperbolic response curve (1) fitted to the measured fluorescence intensity data. A estimates the (background) fluorescence intensity at zero PM-specific spike-in concentration.

$b$ : $\quad$ Saturation fluorescence intensity above the physical background before washing, in a hypothetical situation in which all probes on a feature have formed biotin label carrying duplexes. Assumed to be constant for all features on a microarray.

$B$ : $\quad$ One of the three parameters in the hyperbolic response curve (1) fitted to the measured fluorescence intensity data. $A+B$ estimates the asymptotic saturation fluorescence
$I(x):$
$K$ :
Measured fluorescence intensity signal at the PM-specific spike-in concentration $x$.
One of the three parameters in the
hyperbolic response curve (1) fitted to the measured fluorescence intensity data. $K^{-1}$ estimates the PM-specific spike-in concentration required to give a fluorescence intensity half way between the background level $A$ and asymptotic level $A+B$. the probability that a duplex formed with a PM-feature-specific mRNA target existing at the beginning of the washing step will survive to a washing time $t_{\mathrm{W}}$.
$s^{\mathrm{S}}\left(t_{\mathrm{W}}\right): \quad$ The specific washing survival function, i.e.
$s^{\mathrm{NS}}\left(t_{\mathrm{W}}\right)$ The non-specific washing survival function, i.e. the probability that a duplex formed with a PM-feature-non-specific mRNA target of species $i$ existing at the beginning of the washing step will survive to a washing time $t_{\mathrm{W}}$. concentration. 


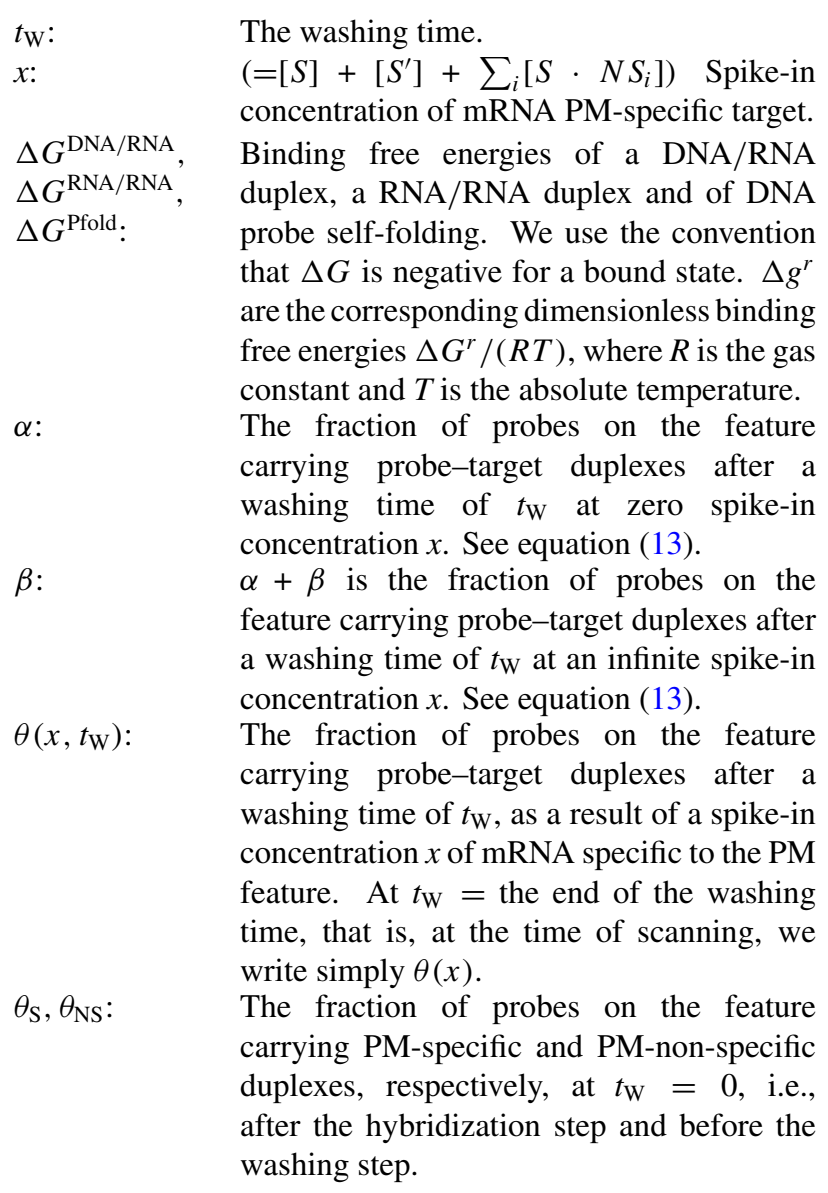

\section{Introduction}

A number of papers [4, 6-8, 10, 14-17] have used chemical adsorption models to analyse data from two well-known Affymetrix Latin-Square spike-in experiments [1], known as the U95 and U133 datasets. The immediate aim of these papers has been to study the physical processes responsible for converting concentrations of specific target RNA in prepared solutions hybridized onto microarrays to measured fluorescence intensities. Their ultimate aim has been to provide biologists with a practical algorithm for estimating absolute specific target concentrations in the presence of a complex non-specific background from the fluorescence intensity data. Even though there are a number of existing algorithms (or 'expression measures') of varying degrees of statistical sophistication currently available to and widely used by biologists, such algorithms pay little attention to the detailed physics and chemistry of hybridization at the microarray surface. As a result they are prone to underestimating fold changes at high target concentrations because saturation effects are not modelled, and at low target concentrations because of a failure to account adequately for non-specific hybridization [9]. Furthermore, the measures reported are not target concentrations as such, but surrogates derived directly from fluorescence intensities. At best, they could be described as an unknown increasing function of specific target concentrations, modulo statistical noise.
Before a reliable algorithm for inferring target concentrations can be developed, an accurate model of the physics and chemistry of the process is needed. In a recent review of chemical adsorption effects at the microarray surface [2], Binder has described in detail a number of processes influencing fluorescence intensity measurements, including bulk dimerization of target molecules in the solution, nonspecific hybridization and probe folding at the microarray surface and partial zippering of duplexes. Analyses of the Affymetrix spike-in data have provided strong evidence that these effects cannot be ignored. For instance, Binder and Preibisch [6] have isolated the effects of specific and nonspecific binding, Carlon and Heim [10] and Heim et al [14] have stressed the importance of bulk hybridization and target folding in the solution, and by analysing other datasets, Matveeva et al [19] have produced evidence for the importance of probe folding.

More recently, analyses of the U95 dataset [8, 17] and subsequent experimental evidence [21] have demonstrated the significant influence of the post-hybridization washing step in determining fluorescence intensities. Although the washing step has an important scaling effect over the whole range of target concentrations, it is particularly noticeable as a probesequence-dependent scaling of the asymptote of the measured intensity isotherm at saturation concentrations. Because adsorption theories of the hybridization step which neglect the post-hybridization washing predict a common asymptote for these isotherms, many adsorption-model-based analyses of the Affymetrix Latin-Square datasets have forced the data to fit a common asymptote $[6,10,14,16]$, in spite of strong statistical evidence to the contrary $[7,15]$.

In this paper, we reanalyse both the U95 and U133 datasets, and a third dataset which is analogous to the U95 dataset, but without the complex background. Our analysis uses a chemical adsorption model which includes a number of chemical reactions occurring during the hybridization and post-hybridization washing steps. Details of the datasets are summarized in section 2, and all the three datasets are shown to be consistent with the empirical observations previously observed for the U95 dataset, namely that measured fluorescence intensities follow a hyperbolic isotherm with probe-sequence-dependent parameters [7]. Our physicochemical model is described in section 3 and demonstrated to be quantitatively consistent with these observations in section 4. A quantitative analysis of how well the model fits the three datasets is given in section 5. An analysis in section 6 gives some indication of how intensity measurements over a whole microarray might be used to infer some of the isotherm parameters for each feature on the microarray, which in turn has the potential to contribute towards the development of a practical algorithm for estimating target concentrations. Concluding comments are given in section 7 .

\section{Datasets and empirical fits}

Three datasets are analysed in this paper, the two publicly available Affymetrix Latin-Square spike-in experiments [1], known as the U95 and U133 datasets, and a version of the 
U95 dataset without the complex human pancreas background, kindly made available to us by Affymetrix. We will refer to these datasets as numbers I, II and III, respectively.

In the manufacture of Affymetrix GeneChip ${ }^{\circledR}$ arrays, single strand DNA probes, 25 bases in length are synthesized base by base onto a quartz substrate using a photolithographic process. They are attached to the substrate via short covalently bonded linker molecules roughly $10 \mathrm{~nm}$ apart. A microarray chip surface is divided into some hundreds or thousands of regions called features, commonly 11-20 $\mathrm{mm}^{2}$, and with the single strand DNA probes within each feature being synthesized to a specific nucleotide sequence.

The main step in the laboratory process of gene detection with microarrays is the hybridization of cRNA target molecules, fractionated to lengths of typically 50-200 bases and with biotin labels attached to their $\mathrm{U}$ and $\mathrm{C}$ bases, onto the single strand DNA probes. The hybridization is performed at $45{ }^{\circ} \mathrm{C}$ for $16 \mathrm{~h}$. The microarray is then washed to remove excess cRNA target, the biotin labels stained with fluorescent dye, and the density of hybridized probe-target duplexes in each feature detected via intensity measurements of the fluorescent dye. Each gene or EST is represented by a set of pairs of features (16 pairs in the case of the U95 dataset and 11 pairs for U133) using sequences of length 25 selected for their predicted hybridization properties and specificity to the target gene. The first element of the pair, termed the perfect match (PM), is designed to be an exact match to the target sequence, while the second element, the mismatch (MM), is identical except for the middle (13th) base being replaced by its complement.

In the Affymetrix spike-in experiments, RNA transcripts were spiked in at cyclic permutations of a set of known concentrations together with a complex background of cRNA extracted from human pancreas (dataset I), human adenocarcinoma cell line (dataset II) or no background (dataset III). Each of the datasets I and III consists of the PM and MM fluorescence intensity values from a set of 14 probesets corresponding to 14 separate genes, each containing 16 probe pairs. For each probeset, a set of fluorescence intensity values was obtained for the 14 spiked-in concentrations $0,0.25,0.5,1,2, \ldots, 1024 \mathrm{pM}$. In common with the previous analyses of dataset I, the following analysis of datasets I and III is restricted to 12 of the 14 genes, omitting data from the two defective probesets, 36889_at and 407_at. Dataset II consists of the PM and MM fluorescence intensity values from a set of 38 probesets corresponding to 38 separate genes, each containing 11 probe pairs. For each probeset, a set of fluorescence intensity values was obtained for the 14 spiked-in concentrations $0,0.125,0.25,0.5,1, \ldots, 512$ pM. Dataset II also contains data from a further 4 bacterial gene probesets each containing 20 probe pairs, which we do not include in the current analysis.

In a previous paper [7], it was demonstrated that the dataset $\mathrm{I}$ is consistent with the empirical observation that the measured fluorescence intensities $I(x)$ at the spike-in concentration $x$ are sampled from a Gamma distribution with a constant coefficient of variation about the mean given by a hyperbolic response curve of the form

$$
I(x)=A+B \frac{K x}{1+K x} .
$$

Importantly, it was further shown using a thorough statistical analysis that each of the parameters $A, B$ and $K$ is feature (i.e. probe-sequence) dependent, and that the asymptote, $\lim _{x \rightarrow \infty} I(x)=A+B$, is almost invariably lower for the $\mathrm{MM}$ feature than the neighbouring perfect-match (PM) feature within any $\mathrm{PM} / \mathrm{MM}$ pair.

On the assumption that by far the greatest proportion of the intensity signal across a whole microarray in either dataset I or II comes from the complex background, we have preprocessed the data by carrying out a quantile normalization across each of these two datasets [18]. Thus, all microarrays within a particular dataset have a common distribution of fluorescence intensities after preprocessing. Because of the absence of a complex background, we have not carried out this step for dataset III. Instead we have included in the fit the 'wafer-dependent scaling' described as Model $\mathbf{E}$ in [7] to allow for the fact that the three replicates of the experiment used microarrays constructed from three separate wafers. That is to say, we fit to the data a model of the form $I(x)=\lambda_{w}\left[A_{p}+B_{p} K_{p} x /\left(1+K_{p} x\right)\right]$, where the index $w=1,2,3$ labels the three replicates, the index $p$ labels a feature within a probeset and the scaling factors satisfy $\frac{1}{3} \sum_{w} \lambda_{w}=1$.

In figures 1 and 2 are plotted the fits of fluorescence intensity data to the response curve (1) for one of the spiked-in transcripts for datasets I and III. A complete set of analogous plots for all transcripts from all the three datasets can be found at http://dayhoff.anu.edu.au/ conrad/Spike_in_Isotherms/.

For a small minority of features the fit gives negative values to some of the parameters $A, B$ or $K$, whereas the physical model set out in section 3 predicts that all the three parameters should be positive. This problem is slightly more prevalent for MM features than for PM features, and is most acute for dataset II. In some cases, such as probe number 3 in figure 1, it appears that the data does not extend far enough into the high concentration, i.e. saturation, limit to allow an acceptable fit. In other cases, such as probe number 9 of figure 2, the data may be too noisy. The range of spike-in concentrations used in dataset II is shifted downwards from that of datasets I and III, and as such may not be adequately probing the saturation region to give acceptable fits in all cases. Furthermore, the spike-in concentrations at the lower end may be probing the regime in which the target concentration is depleted during the hybridization step, which is beyond the applicability of the model leading to equation (1) which we describe below. The analyses in sections 4 and 5 are restricted to the subset of fits to equation (1) for which all the three parameters $A, B$ and $K$ are positive. Table 1 gives the coefficient of variation of the fitted data for each dataset, and the percentage of features for which an acceptable fit with three positive parameters to the hyperbolic response function was obtained. In general, an agreement with a hyperbolic response curve with positive parameters is excellent for datasets I and III, and reasonable for dataset II. 

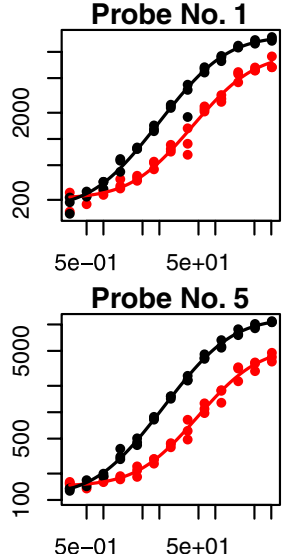

Probe No. 9

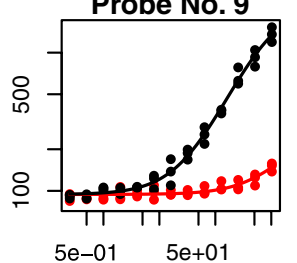

5e-01 5e+01

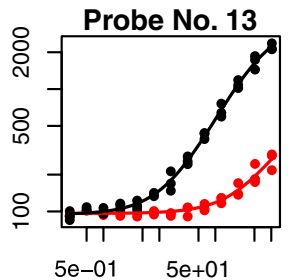

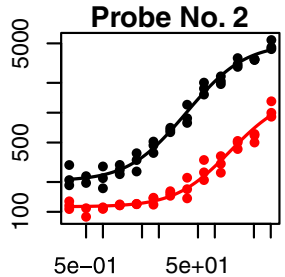

Probe No. 6

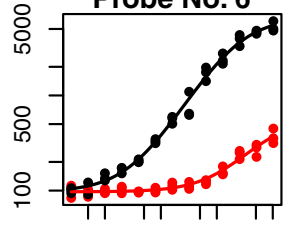

$5 \mathrm{e}-01 \quad 5 \mathrm{e}+01$

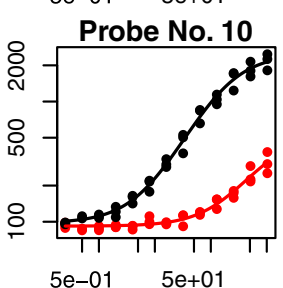

Probe No. 14

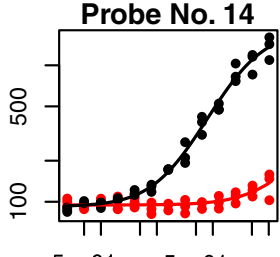

$5 e-01 \quad 5 e+01$

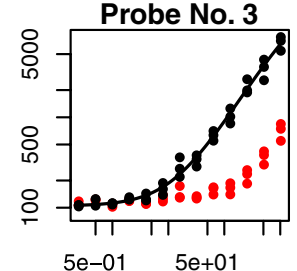

Probe No. 7
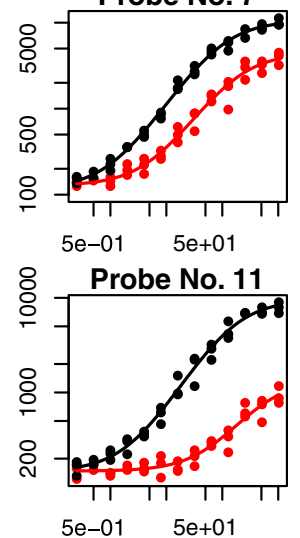

Probe No 15

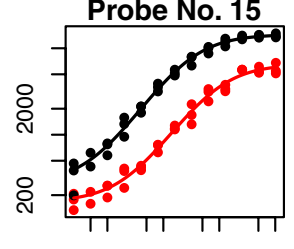

$5 e-01 \quad 5 e+01$

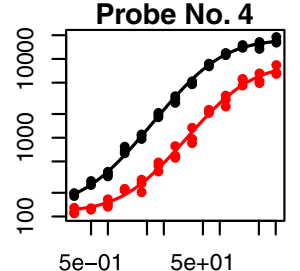

Probe No. 8

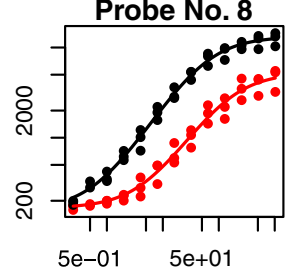

$5 \mathrm{e}-01 \quad 5 \mathrm{e}+01$

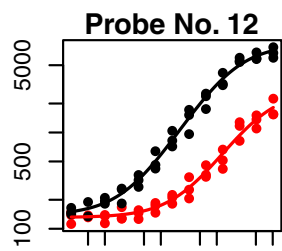

5e-01 5e+01

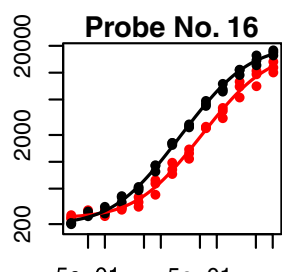

37777_at

Figure 1. Fits of fluorescence intensity data to the hyperbolic response curve (1) for the spiked-in transcript 37777_at for dataset I. PM data are in black and MM data in red (grey). Spike-in concentrations (horizontal axes) in picomolar are on a logarithmic scale, and fluorescence intensities after quantile normalization (vertical axes) are in the arbitrary units used in Affymetrix cell files on a logarithmic scale.

Table 1. The coefficient of variation of the data, assumed to be distributed from a Gamma distribution with a constant coefficient of variation within any one dataset about a mean given by equation (1). The two right-hand columns give the percentage of probesets for which the fit gives positive values to all the three parameters $A, B$ and $K$.

\begin{tabular}{llcl}
\hline \multirow{2}{*}{ Dataset } & \multirow{2}{*}{$\begin{array}{l}\text { Coefficient } \\
\text { of variation }\end{array}$} & \multicolumn{2}{c}{ \% of accepted fits } \\
\cline { 3 - 4 } & PM & MM \\
\hline I & 0.12 & 97.9 & 91.6 \\
II & 0.14 & 72.5 & 37.5 \\
III & 0.17 & 100 & 98.4 \\
\hline
\end{tabular}

\section{The model}

Consider the response of a given feature to the spike-in concentration $x$ of a particular RNA transcript in the presence of an unknown complex background of non-specific target RNA. We write the measured fluorescence intensity $I(x)$ in the form

$$
I(x)=a+b \theta(x),
$$

where $a$ is a physical background due to effects unrelated to fluorescent label carrying duplexes, such as reflection from the glass surface of the microarray, and $b$ is the maximum fluorescence intensity, that is, the contribution from fluorescent dye if all probes on the feature were occupied with labelled probe-target duplexes. It is argued in [2] that the maximum intensity $b$ should vary only weakly due to differing probe sequences. Throughout this paper we assume $a$ and $b$ to be fixed constants for a given microarray. The coverage fraction, $\theta(x)$, is the fraction of probes on the feature carrying probe-target duplexes at the time of scanning. It satisfies $0 \leqslant \theta(x) \leqslant 1$.

The coverage fraction is determined by a number of reactions between various chemical species. The species and reactions considered in our model are set out in tables 2 and 3 , respectively. The first five reactions in table 3 are assumed to reach equilibrium during the hybridization step. The rate constants $K_{i}^{\text {bulk }}, K^{\text {Sfold }}, K^{\mathrm{S}}, K_{i}^{\mathrm{NS}}$ and $K^{\text {Pfold }}$ are the ratio of the forward to backward rates for each reaction. The washing phase, which is primarily designed to remove unbound targets before scanning, also dissociates some duplexes [8, 17]. Thus, the last two reactions are unidirectional as dissociated duplexes are continuously flushed out of the cartridge and replaced with a buffer solution containing no RNA. 

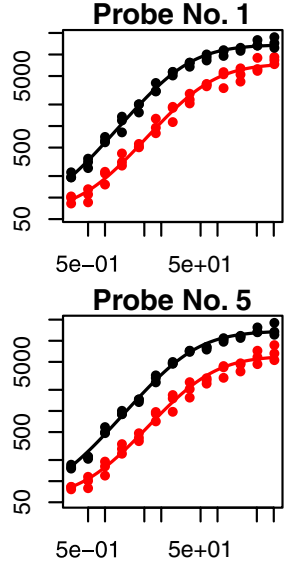

Probe No. 9
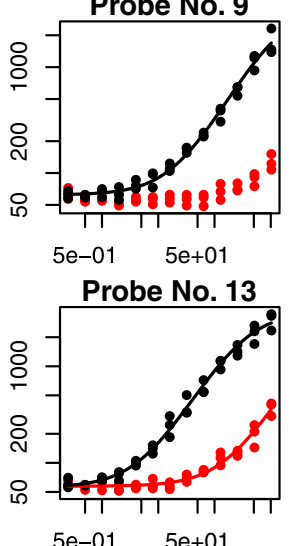
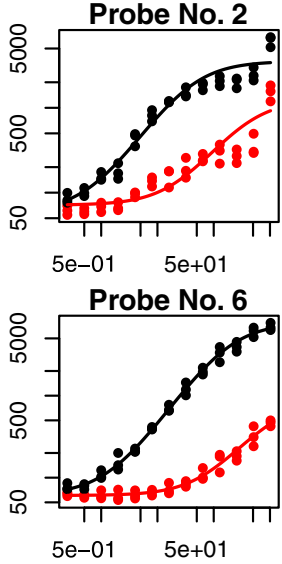

Probe No. 10
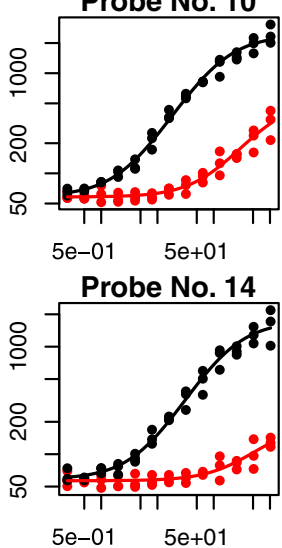
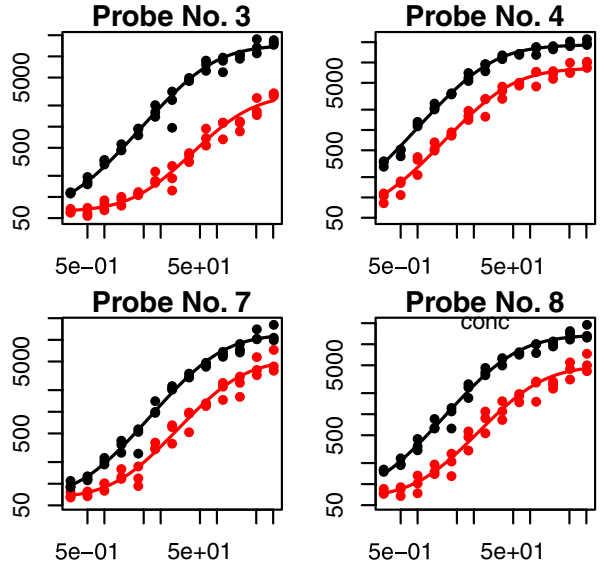

Probe No. 11

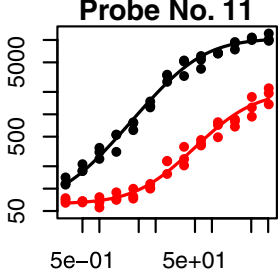

Probe No. 12

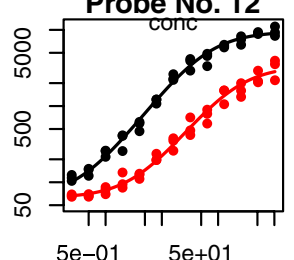

Probe No. 16
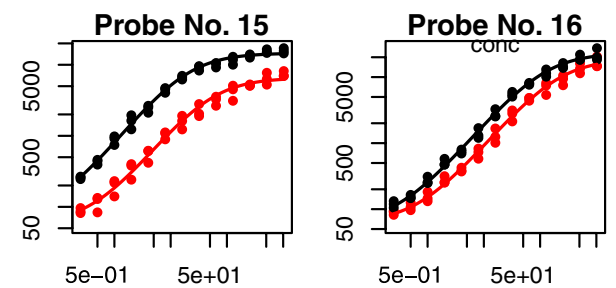

Figure 2. Fits of fluorescence intensity data to the hyperbolic response curve (1) for the spiked-in transcript 37777_at for dataset III, without the complex human pancreas background.

Table 2. Chemical species present in the model.

\begin{tabular}{ll}
\hline$S$ & Single strand PM-feature-specific RNA target in the solution \\
$N S_{i}$ & Single strand non-specific RNA target in the solution of species $i$ \\
$S \cdot N S_{i}$ & Bound RNA/RNA duplex in the solution unavailable for hybridization \\
$S^{\prime}$ & Folded specific target in the solution rendered unavailable for hybridization \\
$P$ & Unbound probe at the microarray surface \\
$P \cdot S$ & Duplex formed from PM-feature-specific RNA target binding to probe \\
$P \cdot N S_{i}$ & Duplex formed from non-specific RNA target binding to probe \\
$P^{\prime}$ & Folded probe at the microarray surface rendered unavailable for hybridization \\
\hline
\end{tabular}

Table 3. Chemical reactions occurring in the model. Rate constants, defined as the ratio of forward to backward reaction rates, are given in the right-hand column.

\begin{tabular}{llll}
\hline In the bulk solution & Non-specific hybridization & $S+N S_{i} \rightleftharpoons S \cdot N S_{i}$ & $K_{i}^{\text {bulk }}$ \\
& Specific target folding & $S \rightleftharpoons S^{\prime}$ & $K^{\text {Sfold }}$ \\
At the microarray & Specific hybridization & $P+S \rightleftharpoons P \cdot S$ & $K^{\mathrm{S}}$ \\
surface & Non-specific hybridization & $P+N S_{i} \rightleftharpoons P \cdot N S_{i}$ & $K_{i}^{\mathrm{NS}}$ \\
& Probe folding & $P \rightleftharpoons P^{\prime}$ & $K^{\text {Pfold }}$ \\
During the & Dissociation of specific duplexes & $P \cdot S \rightarrow P(+S)$ & \\
washing phase & Dissociation of non-specific duplexes & $P \cdot N S_{i} \rightarrow P\left(+N S_{i}\right)$ \\
\hline
\end{tabular}

The effect of the first two reactions, bulk hybridization and specific target folding in the solution, is to reduce the concentration of specific target available for hybridization onto the microarray from its spike-in value of $x$ to a value $[S]$, that is the concentration of single strand RNA target $S$ in the solution. (Following the usual convention, square brackets 
indicate concentrations.) For these reactions we follow the analysis of [14]. The label $i$ in table 3 ranges over all possible subsequences of the 25-mer part of the specific target RNA sequence complementary to the PM probe. The species $N S_{i}$ in this reaction include any target RNA molecule containing a subsequence complementary to the $i$ th subsequence. At equilibrium, we have

$$
\frac{\left[S^{\prime}\right]}{[S]}=K^{\text {Sfold }}, \quad \frac{\left[S \cdot N S_{i}\right]}{[S]\left[N S_{i}\right]}=K_{i}^{\text {bulk }} .
$$

The relation $x=[S]+\left[S^{\prime}\right]+\sum_{i}\left[S \cdot N S_{i}\right]$ then gives

$$
[S]=\frac{x}{1+K^{\text {Sfold }}+X^{\text {bulk }}}
$$

where

$$
X^{\mathrm{bulk}}=\sum_{i}\left[N S_{i}\right] K_{i}^{\mathrm{bulk}}
$$

The following three reactions, occurring at the microarray surface, determine the duplex coverage fraction of the feature at the end of the hybridization step, and before washing. Let the fraction of probes on the feature that have formed duplexes with either specific or non-specific target mRNA molecules and survived to a time $t_{\mathrm{W}}$ after the commencement of the washing process be $\theta\left(x, t_{\mathrm{W}}\right)$. We split the fraction of probes which have formed duplexes at the end of the hybridization step and before washing into two contributions:

$$
\theta(x, 0)=\theta^{\mathrm{S}}+\theta^{\mathrm{NS}} \text {. }
$$

The first contribution, $\theta^{\mathrm{S}} \propto[P \cdot S]$, is that due to duplexes formed with specific mRNA targets, and the second contribution, $\theta^{\mathrm{NS}} \propto \sum_{i}\left[P \cdot N S_{i}\right]$, is that due to duplexes which have formed with non-specific mRNA targets, the sum being over targets containing a subsequence complimentary to the $i$ th subsequence of the probe.

Either by balancing equilibrium concentrations against chemical rate constants [4] or by considering the Gibbs distribution of states at constant chemical potential [8, 13], one obtains the isotherms

$$
\begin{aligned}
\theta^{\mathrm{S}} & =\frac{X^{\mathrm{S}}}{1+K^{\text {Pfold }}+X^{\mathrm{S}}+X^{\mathrm{NS}}}, \\
\theta^{\mathrm{NS}} & =\frac{X^{\mathrm{NS}}}{1+K^{\text {Pfold }}+X^{\mathrm{S}}+X^{\mathrm{NS}}},
\end{aligned}
$$

where, following the notation of [2], we define

$$
X^{\mathrm{S}}=[S] K^{\mathrm{S}}, \quad X^{\mathrm{NS}}=\sum_{i}\left[N S_{i}\right] K_{i}^{\mathrm{NS}} .
$$

The calculation leading to these isotherms assumes a negligible depletion of target molecules in the bulk solution during the hybridization process.

Note that in the asymptotic limit of high spike-in concentration, namely $x \rightarrow \infty$, while holding $\left[N S_{i}\right]$ constant, equations (4)-(9) imply $\theta^{\mathrm{S}} \rightarrow 1$ and $\theta^{\mathrm{NS}} \rightarrow 0$, implying that the feature becomes saturated with specific duplexes. This is contrary to the differing isotherm asymptotes observed empirically. To explain the differing asymptotes, we include in our model the final two reactions in table 3 , namely the washing step [8]. Define the probability that a given probetarget duplex has survived up to a washing time $t_{\mathrm{W}}$ to be $s^{\mathrm{S}}\left(t_{\mathrm{W}}\right)$ for a specific duplex and $s_{i}^{\mathrm{NS}}\left(t_{\mathrm{W}}\right)$ for a non-specific duplex of species $i$. The survival functions $s^{\mathrm{S}}$ and $s_{i}^{\mathrm{NS}}$ depend on the probe and target base sequences, satisfy $s^{\mathrm{S}}(0)=s_{i}^{\mathrm{NS}}(0)=1$, are positive and are monotonically decreasing. Defining an average non-specific survival function $s^{\mathrm{NS}}\left(t_{\mathrm{W}}\right)$ by

$$
X^{\mathrm{NS}} s^{\mathrm{NS}}\left(t_{\mathrm{W}}\right)=\sum_{i}\left[N S_{i}\right] K_{i}^{\mathrm{NS}} s_{i}^{\mathrm{NS}}\left(t_{\mathrm{W}}\right),
$$

the coverage fraction at the washing time $t_{\mathrm{W}}$ is then

$$
\theta\left(x, t_{\mathrm{W}}\right)=\theta^{\mathrm{S}} s^{\mathrm{S}}\left(t_{\mathrm{W}}\right)+\theta^{\mathrm{NS}} s^{\mathrm{NS}}\left(t_{\mathrm{W}}\right) .
$$

Substituting equations (7) and (8) and rearranging gives

$$
\begin{gathered}
\theta\left(x, t_{\mathrm{W}}\right)=\frac{X^{\mathrm{NS}} s^{\mathrm{NS}}\left(t_{\mathrm{W}}\right)}{1+K^{\text {Pfold }}+X^{\mathrm{NS}}}+\left(s^{\mathrm{S}}\left(t_{\mathrm{W}}\right)-\frac{X^{\mathrm{NS}} s^{\mathrm{NS}}\left(t_{\mathrm{W}}\right)}{1+K^{\text {Pfold }}+X^{\mathrm{NS}}}\right) \\
\times \frac{\left(1+K^{\text {Pfold }}+X^{\mathrm{NS}}\right)^{-1} X^{\mathrm{S}}}{1+\left(1+K^{\text {Pfold }}+X^{\mathrm{NS}}\right)^{-1} X^{\mathrm{S}}} .
\end{gathered}
$$

Finally, with the help of equations (4) and (9), and suppressing the $t_{\mathrm{W}}$ dependence, we get

$$
\theta(x)=\alpha+\beta \frac{K x}{1+K x}
$$

where

$$
\begin{gathered}
\alpha=\frac{X^{\mathrm{NS}} s^{\mathrm{NS}}}{1+K^{\text {Pfold }}+X^{\mathrm{NS}}}, \\
\beta=s^{\mathrm{S}}-\alpha, \\
K=\frac{K^{\mathrm{S}}}{\left(1+K^{\text {Sfold }}+X^{\text {bulk }}\right)\left(1+K^{\text {Pfold }}+X^{\mathrm{NS}}\right)} .
\end{gathered}
$$

This model, summarized by equations (2) and (13), is consistent with the empirical observation of equation (1) with

$$
A=a+b \alpha, \quad B=b \beta .
$$

Equations (14)-(17) (with the help of equations (5), (9) and (10)) relate the empirically fitted parameters $A, B$ and $K$ to underlying physical quantities, namely $a, b$, the concentrations of chemical species in table 2 , reaction rates in table 3 and washing survival functions $s^{\mathrm{S}}$ and $s_{i}^{\mathrm{NS}}$.

Physical effects which have not been included in the model include target depletion, which should only manifest at extremely low target concentrations, incomplete probe synthesis during the manufacturing process [12] and probeprobe interactions. Each of these effects will, in theory, cause the response curve to deviate from a hyperbolic form. A discussion of probe-probe interactions, for instance, can be found in [8]. The choice of model in this paper is guided by a desire to balance the complexity of the problem with practicality.

\section{Qualitative behaviour of the fits}

Before considering a detailed analysis of the ability of the model to explain the parameters of the empirical fits, one can carry out a number of simple qualitative checks. The first three panels of figure 3 compare the fitted saturation asymptotes $A+B$ for PM/MM pairs of features for each of the three datasets. Consistent with the hypothesis that a portion of the bound probe-target duplexes are removed during the washing 

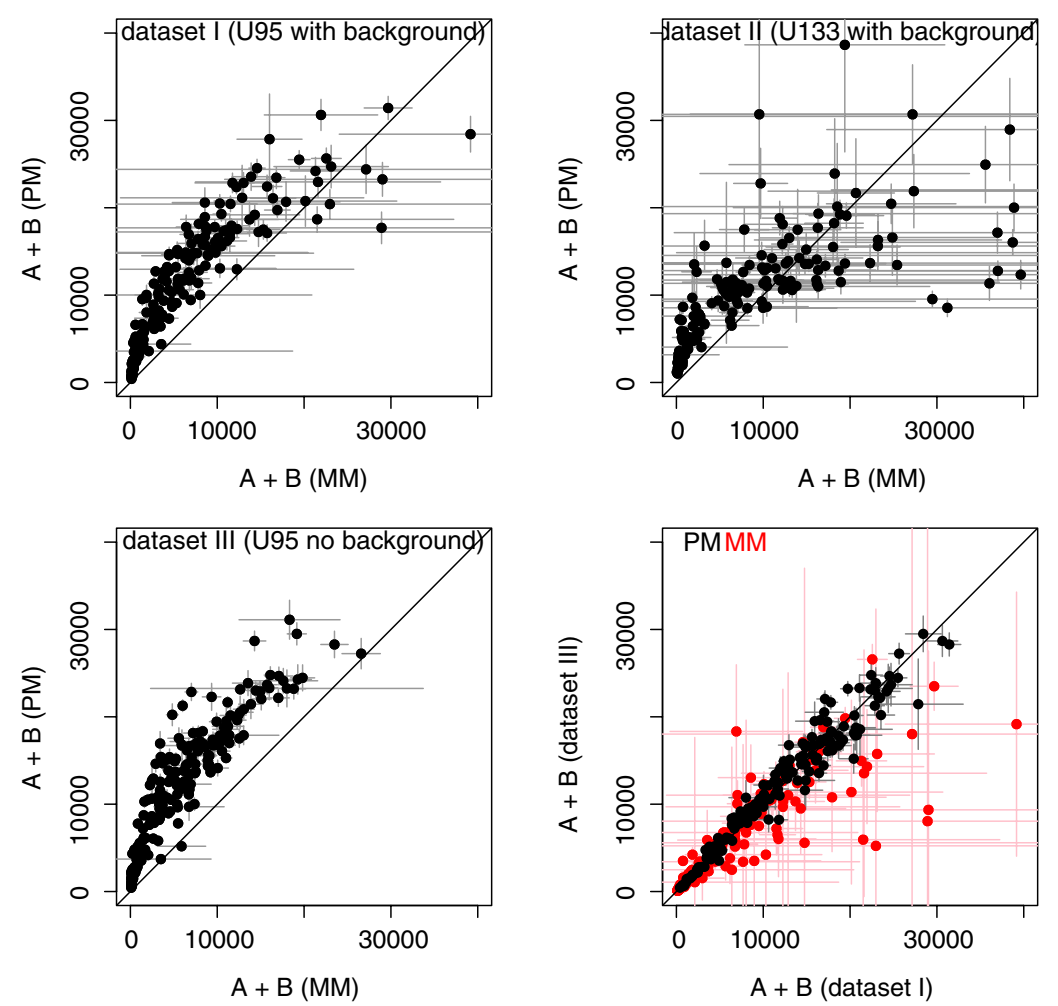

Figure 3. The first three panels show fitted asymptotes $I(\infty)=A+B$, defined in equation (1) for PM/MM pairs for each of the three datasets. The fourth panel compares the asymptotes for dataset I (with non-specific background) with those for dataset III (without non-specific background). Standard errors arising from the fits to equation (1) are also shown.

step, the asymptotes cover a broad range of values. The MM asymptote is almost always less than its PM partner, consistent with the scenario that a saturated feature of PM duplexes will lose less duplexes to washing than the partner saturated feature of less tightly bound MM duplexes [8]. The observed pattern breaks down at higher values of $A+B$, as the fits must be extrapolated further past the highest spike-in concentration to estimate the asymptote, and numerical accuracy is lost. This is most evident for dataset II, for which spike-in concentrations only extend up to $512 \mathrm{pM}$, compared with $1024 \mathrm{pM}$ for datasets I and III.

From equations (15) and (17), the saturation asymptote is given by $I(\infty)=A+B=a+b s^{\mathrm{S}}$. This depends only on the rate $s^{S}$ at which specific duplexes are dissociated by the washing process, and not on the properties of non-specific duplexes. Thus, the model predicts that the asymptote of the response function is unaffected by non-specific hybridization. The fourth panel of figure 3, which compares the asymptote for dataset I (U95 with a complex non-specific background), with that for dataset III (U95 without a non-specific background), confirms this. That is, the asymptote $I(\infty)$ for any feature is the same for dataset I as for dataset III to within the standard errors of the isotherm fits.

The parameter $A$ in equation (1) is the baseline intensity estimate at zero spike-in concentration. From equation (17), it consists of a physical background component $a$, and a component due to non-specific hybridization, $b \alpha$. Consistent with this, the $A$ values, shown in figure 4 , are spread over a much broader range for datasets I and II in which a complex mRNA background was present than for dataset III with no background and therefore little non-specific hybridization.

An obvious pattern, which emerges from comparing $A$ with PM/MM pairs of features in datasets I and II, is that nonspecific hybridization is stronger for a probe whose middle base is a pyrimidine ( $\mathrm{C}$ or $\mathrm{T}$ ) than for its partner probe whose middle base is a purine (A or G). This effect has been noted previously for microarray intensity data generally, and there is some debate about its physical origins $[5,11,20]$. The effect is better understood at the level of individual letter frequencies. Binder et al [4] have noted that probe sensitivities increase with the C-content, and decrease with the A-content, while the Gor T-content of the probe has a little effect. There are probably two effects contributing to this pattern. First, the averaged contributions to DNA/RNA binding energies calculated from nearest-neighbour stacking models [22] are ordered as [3, 11]

$$
\left|\Delta G_{\mathrm{C}}^{\mathrm{av}}\right|>\left|\Delta G_{\mathrm{G}}^{\mathrm{av}}\right| \approx\left|\Delta G_{\mathrm{T}}^{\mathrm{av}}\right|>\left|\Delta G_{\mathrm{A}}^{\mathrm{av}}\right|,
$$

causing the substitution of a pyrimidine by a purine to decrease the probe sensitivity and vice versa. Second, there is a simple geometric effect that pyrimidines, having a small single ring structure, will tolerate mismatches more easily than purines, which have a double ring structure, and would therefore need to deform the molecular backbone to bind with a target closely matching the probe sequence on either side of the central base. No obvious pyrimidine/purine asymmetry is observed in the $A$ values from dataset III. This is to be expected as the parameter 

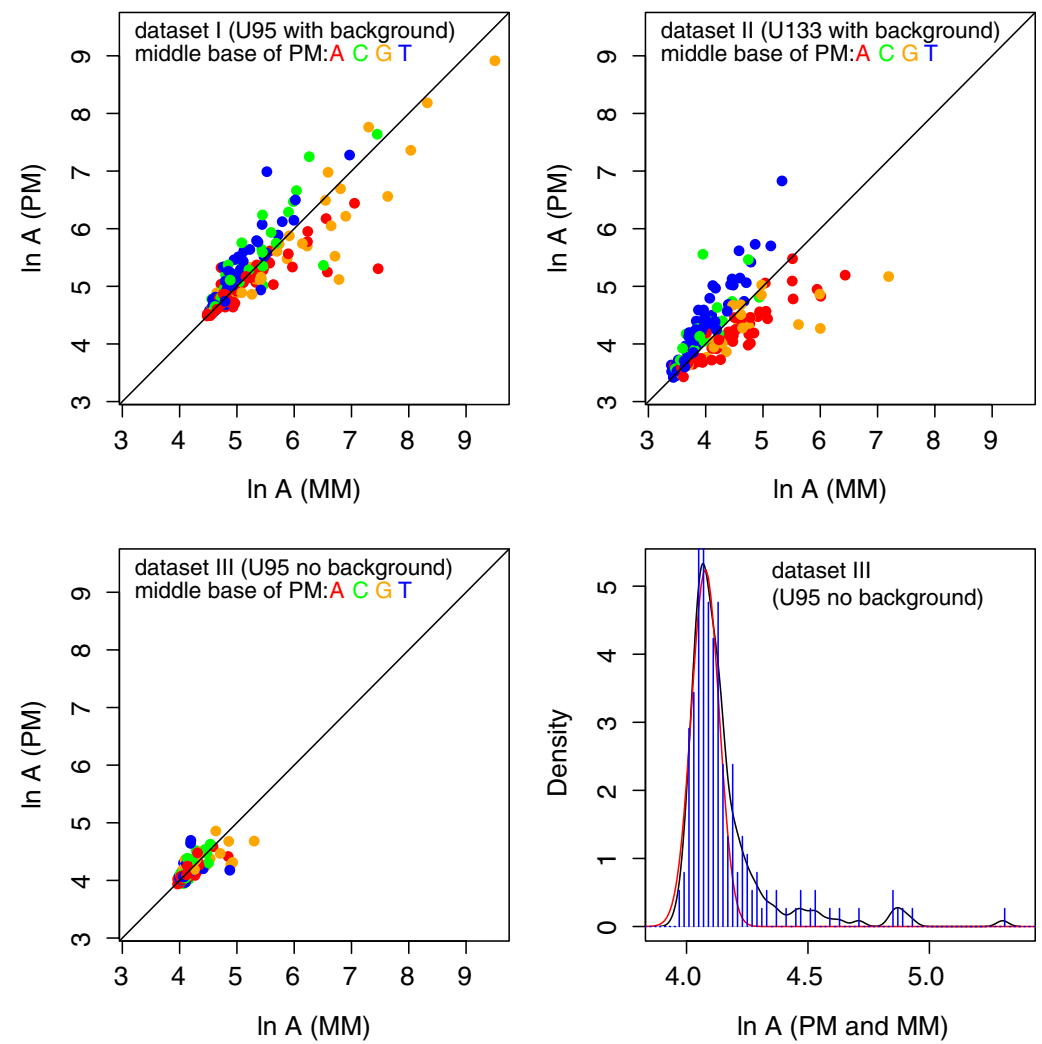

Figure 4. The $\log$ of the fitted baseline fluorescence intensities $I(0)=A$, defined in equation (1), for PM/MM pairs for datasets I and III. The middle (13th) base of the PM probe sequence is colour coded as indicated. The fourth panel shows a histogram of $\ln A$ (in blue), a kernel density estimate of the histogram using a Gaussian kernel with a bandwidth of 0.025 (in black), and a fit of the left part of the histogram to a Gamma distribution in $A$ with a mean of 59 and a coefficient of variation of 0.057 (in red).

$A$ in this case is essentially the physical background without hybridization contributions.

The fourth panel of figure 4 shows a histogram of $A$ values from dataset III, for which there is no complex background present. There is very little non-specific hybridization, and most of these data represent statistical noise in the physical background parameter $a$ (defined in equation (2)). Ignoring the tail, which we assume to be due to a small amount of non-specific hybridization from the other spiked-in transcripts in the Latin-Square protocol, we estimate $a$ by fitting the lefthand part of the histogram to a Gamma distribution in the unlogged data. The fitted distribution has a mean of 59 and a coefficient of variation of 0.057 .

Various comparisons of the effective adsorption rate constant $K$ are shown in figure 5. This parameter is modelled in terms of more fundamental rate constants via equation (16). One can reasonably assume the differences between PM and MM for the indirect effective rate constants $X^{\text {bulk }}, K^{\text {Pfold }}$ and $X^{\mathrm{NS}}$ to be small compared with that for the specific rate constant $K^{\mathrm{S}}$, because of averaging over large numbers of non-specific species. Thus, to a reasonable approximation, $\ln K_{\mathrm{PM}} / K_{\mathrm{MM}} \approx \ln K_{\mathrm{PM}}^{\mathrm{S}} / K_{\mathrm{MM}}^{\mathrm{S}} \approx-\Delta \Delta G / R T$, where $\Delta \Delta G$ is the difference in specific binding energies between a specific mRNA target and a PM and MM probe, respectively. This empirical result has been noted previously as a shift away from the diagonal in a plot of $K_{\mathrm{PM}}$ versus $K_{\mathrm{MM}}$ for dataset I
Table 4. $-\Delta \Delta G$ estimated from a weighted average of $\left(\ln K_{\mathrm{PM}}-\ln K_{\mathrm{MM}}\right)$ for each of the four central bases.

\begin{tabular}{lllll}
\hline & C & G & T & A \\
\hline Dataset I & $1.09 \pm 0.05$ & $0.94 \pm 0.06$ & $0.98 \pm 0.04$ & $0.74 \pm 0.04$ \\
Dataset II & $1.74 \pm 0.14$ & $1.43 \pm 0.10$ & $1.26 \pm 0.05$ & $0.84 \pm 0.06$ \\
Dataset III & $1.10 \pm 0.04$ & $0.90 \pm 0.04$ & $0.97 \pm 0.03$ & $0.66 \pm 0.03$
\end{tabular}

[15] and dataset II [6], and is confirmed here for all the three datasets.

Estimates of $-\Delta \Delta G$ were obtained by taking a weighted average of $-\Delta \Delta G_{p}=\left(\ln K_{\mathrm{PM}}-\ln K_{\mathrm{MM}}\right)_{p}$ over fitted isotherms $p$ for each of the central base letters. The weighting $\sum_{p}\left(-\Delta \Delta G_{p} / s_{p}^{2}\right) / \sum_{p}\left(1 / s_{p}^{2}\right)$, where $s_{p}$ is the standard error in $\Delta \Delta G_{p}$ estimated from the standard error in $K_{p}$ arising from the isotherm fit, is chosen to minimize the error in the average. The results, given in table 4, are consistent with the ordering of binding energies calculated from nearestneighbour stacking models in equation (18).

The third panel of figure 5 compares the effective rate constant $K$ for the U95 experiments with and without the complex human pancreas background. From equation (16), one sees that removing the background, which has the effect of setting $X^{\text {bulk }}$ and $X^{\mathrm{NS}}$ to zero, should increase $K$. This is confirmed in the plot, and is also evident as a shift in the inflection points to the left between figures 1 and 2 . The 

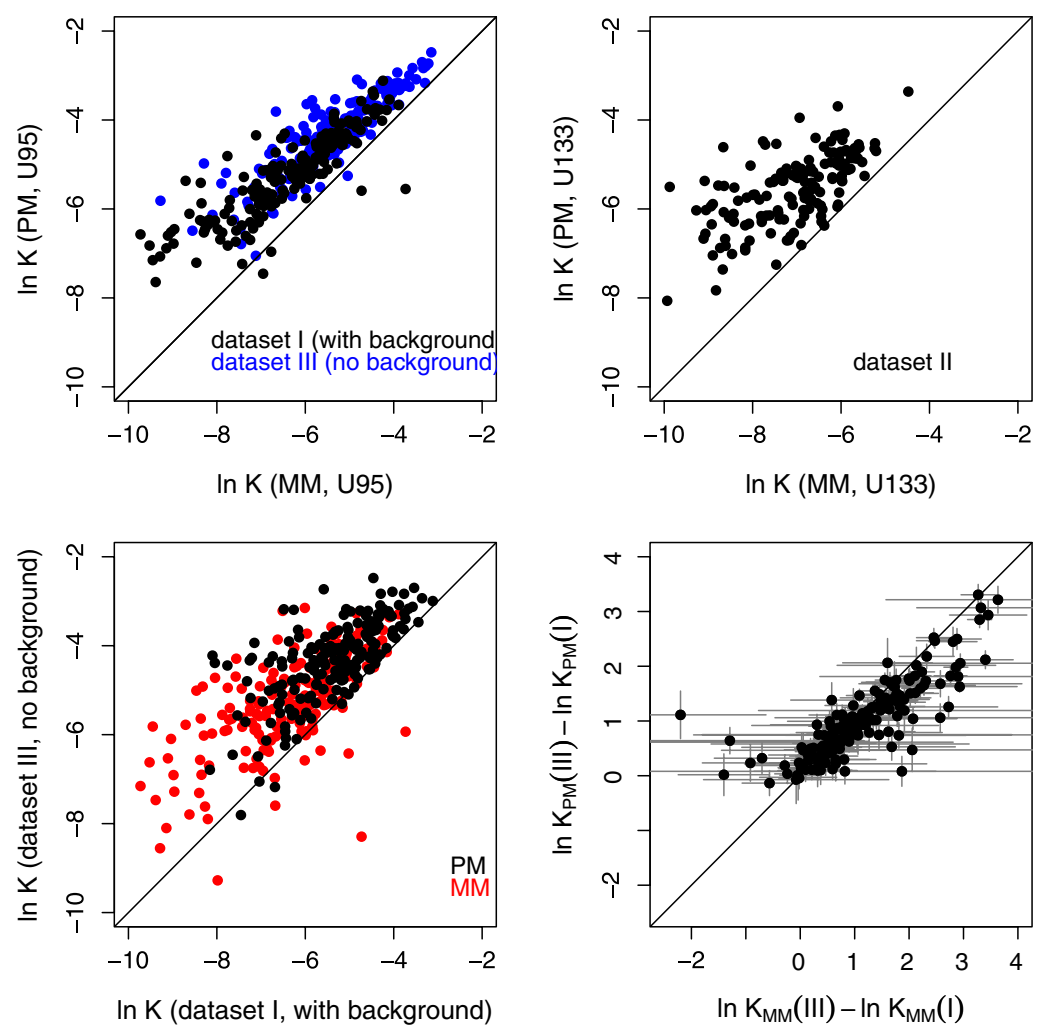

Figure 5. The log of the fitted parameter $K$, defined in equation (1). The first and second panels compare PM/MM pairs for each of the three datasets. The third panel compares datasets I and III, with and without the complex human pancreas background, respectively. The fourth panel compares the increase in $\ln K$ for MM with that for PM when the complex human pancreas background is removed.

fourth panel compares the amount by which $\ln K$ increases as the complex background is removed for PM and MM. We observe that removing the effects of $X^{\text {bulk }}$ and $X^{\mathrm{NS}}$ affects $K$ for a PM probe and its MM partner by a similar factor. The small number of points away from the diagonal line to the left of the plot are caused by the difficulty in fitting the MM isotherm when $K_{\mathrm{MM}}^{-1}$ is beyond the upper limit of the range of spike-in concentrations.

\section{Quantitative behaviour of the fits}

In this section, we explore the ability of the model to explain the quantitative relationship between the fitting parameters of the hyperbolic response curve (1) and known physical properties of microarrays. We divide the analysis into two parts: the parameters $A$ and $B$ which set the vertical scale of the hyperbolic response curve, and the parameter $K$ which sets its horizontal scale.

\subsection{The vertical scale parameters}

The parameters $A$ and $B$ are explained in the model in terms of the more fundamental quantities $a$, the physical background and $b$, saturation intensity above background (which together set the intensity scale in terms of the dimensionless duplex coverage fraction) and $\alpha$ and $\beta$ (which are driven by the chemical reactions in table 3 ).
We begin with $a$ and $b$, which are assumed to be fixed for an entire microarray. In figure 6 are plotted histograms of measured fluorescence intensities across microarrays from datasets I and II. Because these data have been quantile normalized, a common histogram will apply to all microarrays within a given dataset. In the absence of statistical noise, the parameters $a$ and $a+b$ should provide bounding limits for these histograms. However, given the coefficients of variation reported in table 1 , the raw intensity measurements could well extend beyond these limits. According to equation (17), the parameter $a$ should also be a lower cutoff on the fitted parameter $A$ (corresponding to the case of negligible nonspecific hybridization), while the analysis of $A$ in section 4 for dataset III (see the fourth panel of figure 4) suggests a much smaller coefficient of variation for the fitted value of $A$ than for the intensity data generally. For these reasons we use as an estimate of $a$ the minimum over all fits within a dataset of the parameter $A$. These estimates are shown in figure 6, together with bars extending two standard deviations on either side using the coefficients of variation in table 1.

To estimate the saturation parameter $b$ from fits to the hyperbolic response function, and to explain the observed values of the combination $\alpha+\beta$, we make use of the model's prediction that the asymptotic intensity at high spikein concentration, $I(\infty)$, is determined by the washing-step survival function of PM-specific duplexes, $s^{\mathrm{S}}\left(t_{\mathrm{W}}\right)$ [8]. Thus, 

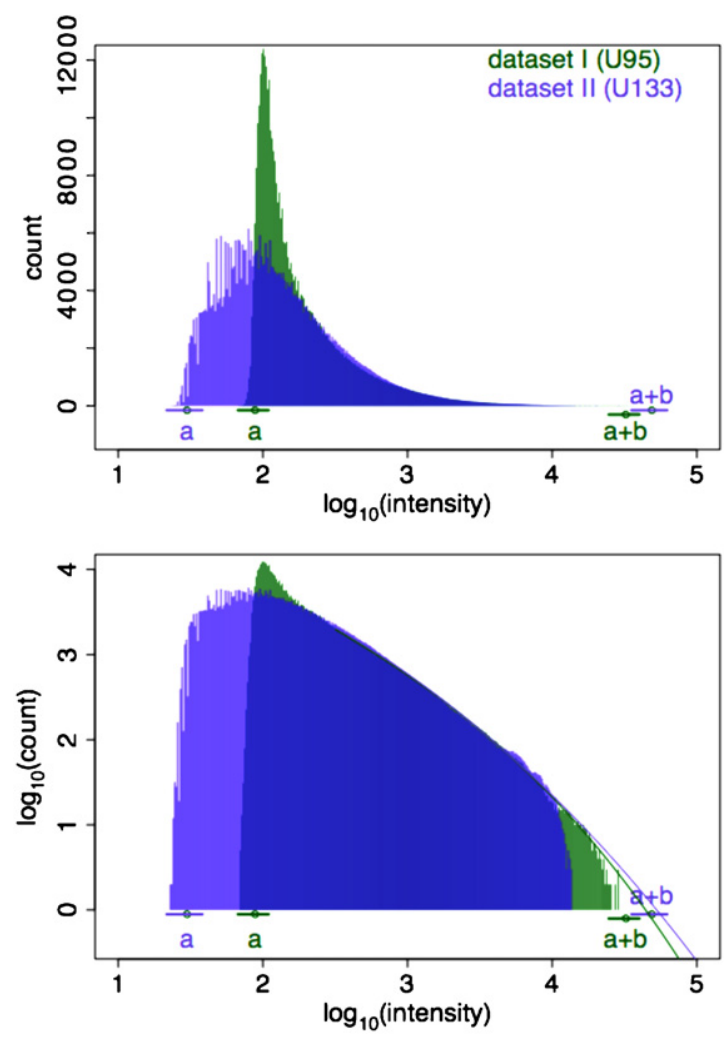

Figure 6. Histograms of quantile normalized fluorescence intensities across microarrays in datasets I and II on a linear (upper) and logarithmic (lower) scale. Both PM and MM intensities are included. Counts are from bins of size 0.01 on the log intensity axis. Also indicated are estimates of the parameters $a$ and $b$ for each dataset, with bars indicating two standard deviations of the spread in the intensity data on either side. The curves fitted to the histograms in the lower panel are explained in section 6.

from equations (15) and (17) we have

$I(\infty)=A+B=a+b(\alpha+\beta)=a+b s^{\mathrm{S}}\left(t_{\mathrm{W}}\right)=a+b \mathrm{e}^{-\kappa t_{\mathrm{W}}}$,

where we assume a uniform washing rate $\kappa$ that depends only on the probe and target nucleotide sequences via their binding

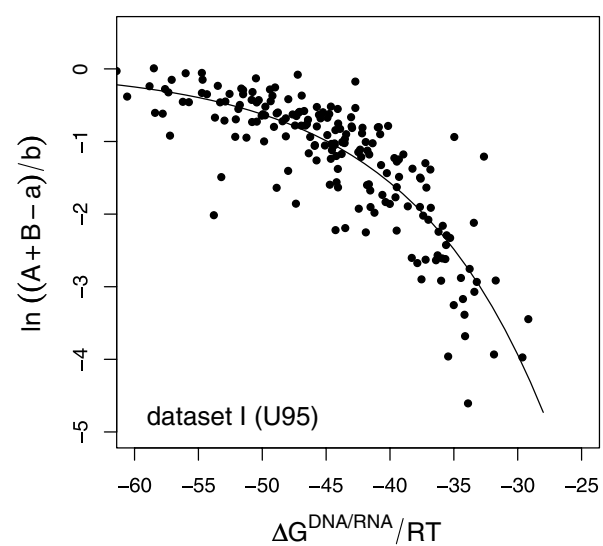

Table 5. Fitted parameters, to three significant figures, occurring in the analysis of section 5 .

\begin{tabular}{llclc}
\hline $\begin{array}{l}\text { Defining } \\
\text { equation }\end{array}$ & Parameter & Dataset I & Dataset II & Dataset III \\
\hline$(2)$ & $a$ & 88.4 & 30.0 & \\
& $\log _{10} a$ & 1.95 & 1.48 & \\
& $b$ & 32300 & 48800 & \\
$(20)$ & $\log _{10} b$ & 4.51 & 4.69 & \\
& $c_{0}$ & 62.2 & 37.9 & \\
$(24)$ & $\lambda_{0}$ & 0.0920 & 0.0841 & \\
$(\alpha$ Model 2) $)$ & $c_{1}$ & -16.1 & -14.8 & \\
& $c_{2}$ & -0.186 & -0.148 & \\
$(28)$ & $c_{3}$ & 0.124 & 0.102 & \\
$(\alpha$ Model 5) $)$ & $c_{1}$ & -14.8 & -14.8 & \\
& $c_{2}$ & -0.200 & -0.149 & \\
& $c_{3}$ & 0.0776 & 0.101 & \\
$(43)$ & $\lambda_{\alpha}$ & 0.176 & 0.909 & \\
$(K$ Model 7) & $\mu_{\alpha}$ & 4.57 & -6.14 & \\
& $\lambda_{\mathrm{S}}$ & 0.145 & 0.0824 & 0.0944 \\
& $\lambda_{\text {Sfold }}$ & -62.9 & 69.4 & -73.4 \\
& $\mu_{\text {Sfold }}$ & -59.3 & -51.5 & -73.7 \\
& $\lambda_{\text {Pfold }}$ & 0.268 & 0.100 & 0.385 \\
& $\mu_{\text {Pfold }}$ & -0.757 & 136 & 0.917 \\
\hline
\end{tabular}

affinity. Following [8], we model $\kappa$ in terms of the RNA/DNA duplex free binding energy in the bulk solution:

$$
\kappa t_{\mathrm{W}}=c_{0} \mathrm{e}^{\lambda_{0} \Delta G^{\mathrm{DNA} / R N A} /(R T)} .
$$

Here, $\Delta G^{\mathrm{DNA} / \mathrm{RNA}}$ is calculated using the nearest-neighbour stacking model and parameters of [22], $R$ is the gas constant, $T$ is the absolute temperature and $c_{0}$ and $\lambda_{0}$ are undetermined constants. We use the convention that $\Delta G^{\mathrm{DNA} / \mathrm{RNA}}$ is negative for a bound state. Rearranging gives

$$
\ln (\alpha+\beta)=\ln \frac{A+B-a}{b}=-c_{0} \mathrm{e}^{\lambda_{0} \Delta G^{\mathrm{DNA} / \mathrm{RNA}} /(R T),}
$$

where $A$ and $B$ are determined for each feature from fitted hyperbolic response functions, $a$ has been estimated above, and $\ln b, c_{0}$ and $\lambda_{0}$ are fitting parameters. Fits to datasets I and II are shown in figure 7 , and fitting parameters are listed in table 5. The fits were done by minimizing the sum of the squares of the residuals with respect to the three fitting parameters.

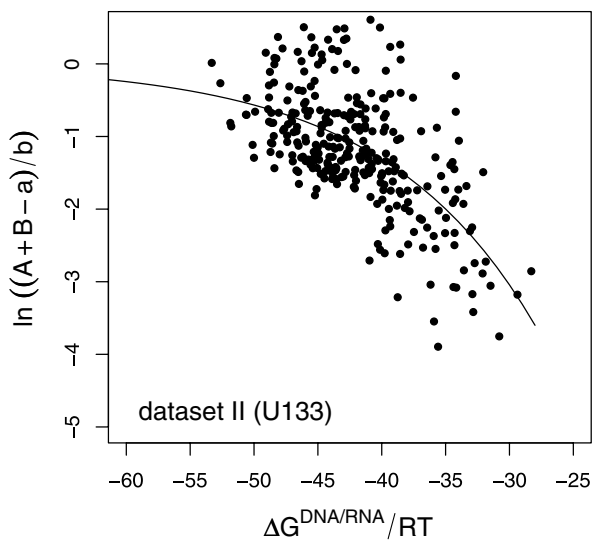

Figure 7. Fits of equation (21) to the parameter combination $A+B$ of the hyperbolic response function fits to the PM data for datasets I and II. The parameter $b$ has been absorbed into a shift in the ordinate. 
The parameter $\alpha$ is in principle predicted by the model in terms of fundamental physical constants via equations (10) and (14). It is determined mainly by non-specific hybridization, including a strong influence from the probe sequence pyrimidine content as suggested in figure 4 , and by probe folding. Without a detailed knowledge of the composition of the non-specific target solution, a direct evaluation of $\alpha$ is of course impossible. However, one can aim for an ansatz in terms of those quantities which are known. Following the reasoning used above, the washing-step survival function of the $i$ th non-specific species $s_{i}^{\mathrm{NS}}\left(t_{\mathrm{W}}\right)$ can be assumed to take the form of a double exponential function of the corresponding free binding energy $\Delta G_{i}^{\mathrm{DNA} / \mathrm{RNA}}$. The value of the double exponential function $\left(f(x)=\mathrm{e}^{-\mathrm{e}^{x}}\right)$ undergoes a changeover from 1 for $x \ll 0$ to 0 for $x \gg 0$ over a narrow range of its argument. Thus, the factor $s_{i}^{\mathrm{NS}}\left(t_{\mathrm{W}}\right)$ in equation (10) can be thought of as a switch which removes from the sum any binding configuration $i$ less tightly bound than some threshold.

The numerator of equation (14) is then a sum of reaction rate constants $K_{i}^{\mathrm{NS}}$, weighted by the number of target molecules in solution with nucleotide sequences complementary to the $i$ th subsequence of the probe. Numerical estimates carried out in the context of bulk hybridization in solution [14] show that such a sum can be well approximated as an exponential function of free binding energy of the entire probe sequence to its complement. Assuming then that the behaviour of $\alpha$ is dominantly exponential in $\Delta G^{\mathrm{DNA} / \mathrm{RNA}}$, and taking into account the added effect of the probe's pyrimidine count, we have tested the following nested models:

Model 0: $\ln \alpha=c_{1}+\epsilon$,

Model 1: $\ln \alpha=c_{1}+c_{2} \Delta g^{\mathrm{DNA} / \mathrm{RNA}}+\epsilon$,

Model 2: $\ln \alpha=c_{1}+c_{2} \Delta g^{\mathrm{DNA} / \mathrm{RNA}}+c_{3} n_{\mathrm{pyr}}+\epsilon$,

Model 3: $\ln \alpha=c_{1}+c_{2} \Delta g^{\mathrm{DNA} / \mathrm{RNA}}+c_{3} n_{\mathrm{pyr}}$

$$
+c_{4} n_{\mathrm{pyr}} \Delta g^{\mathrm{DNA} / \mathrm{RNA}}+\epsilon \text {, }
$$

where $c_{1}, \ldots, c_{4}$ are fitting parameters to be determined, $\Delta g^{\mathrm{DNA} / \mathrm{RNA}}=\Delta G^{\mathrm{DNA} / \mathrm{RNA}} /(R T), n_{\mathrm{pyr}}$ is a count of the number of pyrimidines in the 25-mer probe sequence and $\epsilon$ is the residual error, which is assumed Gaussian.

To include the effect of probe folding, we approximate $K^{\text {Pfold }}$ in equation (14) by a single exponential term

$$
K^{\text {Pfold }}=\exp \left[\lambda_{\alpha}\left(\mu_{\alpha}-\Delta g^{\text {DNA-fold }}\right)\right]
$$

where $\lambda_{\alpha}$ and $\mu_{\alpha}$ are fitting parameters and $\Delta g^{\text {DNA-fold }}=$ $\Delta G^{\mathrm{DNA}-\text { fold }} /(R T)$, where $\Delta G^{\mathrm{DNA}-\text { fold }}$ is calculated for each 25-mer probe sequence from Zuker's Mfold web server [24] with the temperature set to $45^{\circ} \mathrm{C}$ and other parameters set to their default values. The Mfold web server calculates the free energy of the most energetic folding configuration of a given single strand DNA sequence, though ideally one should include a Boltzmann weighted sum over all possible folding configurations. Models 1 and 2 are then nested within Models 4 and 5, respectively:

$$
\begin{aligned}
& \text { Model 4: } \ln \alpha=c_{1}+c_{2} \Delta g^{\mathrm{DNA} / \mathrm{RNA}} \\
& -\ln \left\{1+\exp \left[\lambda_{\alpha}\left(\mu_{\alpha}-\Delta g^{\mathrm{DNA}-\text { fold }}\right)\right]\right\}+\epsilon,
\end{aligned}
$$

Table 6. $p$-Values testing significance of the extra parameter related to nested pairs of models in equations (22)-(28). Smaller $p$-values indicate that the extra parameters in the more complicated model are significant. The second column gives the extra parameters included in the more complicated of the two models.

\begin{tabular}{llll}
\hline & Parameter & Dataset I & Dataset II \\
\hline Model 0 to Model 1: & $c_{2}$ & $<2 \times 10^{-16}$ & $<2 \times 10^{-16}$ \\
Model 1 to Model 2: & $c_{3}$ & $1.2 \times 10^{-9}$ & $1.9 \times 10^{-6}$ \\
Model 2 to Model 3: & $c_{4}$ & 0.0098 & 0.648 \\
Model 1 to Model 4: & $\lambda_{\alpha}, \mu_{\alpha}$ & $1.1 \times 10^{-10}$ & 0.60 \\
Model 2 to Model 5: & $\lambda_{\alpha}, \mu_{\alpha}$ & $3.4 \times 10^{-5}$ & 0.81 \\
Model 4 to Model 5: & $c_{3}$ & 0.00064 & $2.7 \times 10^{-6}$ \\
\hline
\end{tabular}

Model 5: $\ln \alpha=c_{1}+c_{2} \Delta g^{\mathrm{DNA} / \mathrm{RNA}}+c_{3} n_{\mathrm{pyr}}$

$$
-\ln \left\{1+\exp \left[\lambda_{\alpha}\left(\mu_{\alpha}-\Delta g^{\text {DNA-fold }}\right)\right]\right\}+\epsilon \text {. }
$$

The above nested models can be tested for the significance of the extra parameters introduced in going from a less to a more complicated model. For instance, to test the significance of the extra parameter distinguishing model $m_{2}$ from the simpler $m_{1}$, consider for each model the residual sums of squares $D=\sum \epsilon^{2}$, where the sum is taken over fitted data points. Under the null hypothesis that the extra complexity is not significant, and assuming the data points to be independent, the test statistic defined by

$$
F=\frac{\left(D_{1}-D_{2}\right) /\left(d_{1}-d_{2}\right)}{D_{2} / d_{2}}
$$

(where $d_{1}$ and $d_{2}$ count the residual degrees of freedom of $m_{1}$ and $m_{2}$, respectively) has an $F$ distribution with degrees of freedom equal to $d_{1}-d_{2}$ and $d_{2}$. This allows us to assign a $p$-value to the significance of model $m_{2}$ over $m_{1}$.

We have fitted each of the five models to the combination $\alpha=(A-a) / b$ using $a$ and $b$ from table 5 and $A$ from the hyperbolic response function fits of both PM and MM data for datasets I and II separately. The number of data points fitted, that is, the number of fitted hyperbolic response functions for which all the three parameters $A, B$ and $K$ are positive, was 364 for dataset I and 460 for dataset II. Table 6 gives the calculated $p$-values.

The parameters $c_{2}$ and $c_{3}$ modelling linear effects in $\Delta G^{\mathrm{DNA} / \mathrm{RNA}}$ and $n_{\mathrm{pyr}}$, respectively, are highly significant in both datasets. The parameter $c_{4}$ defining a mixed effect is barely significant at the $1 \%$ level in dataset I and not significant in dataset II, and we shall ignore it from here on.

The probe-folding effect is highly significant for dataset I, but not significant for dataset II. Note that Models 4 and 5 contain the functional form

$-\ln \{1+\exp [\lambda(\mu-\Delta g)]\} \approx \begin{cases}\lambda(\Delta g-\mu), & \Delta g \ll \mu, \\ 0, & \Delta g \gg \mu,\end{cases}$

for $\lambda>0$. Thus, the probe-folding effect 'switches on' once the energy of a folded probe is below some threshold $\mu_{\alpha}$, at which point the effect becomes linear in $\Delta g^{\text {DNA-fold }}$. From table 5 , the fitted value of $\mu_{\alpha}$ in Model 5 for dataset $\mathrm{I}$ is 4.57, whereas the range of probe-folding energies calculated by Mfold for the probe sequences of the U95 microarray is $-8.18<\Delta g^{\text {DNA-fold }}<2.98$. Thus, $\mu_{\alpha}$ is well above the 

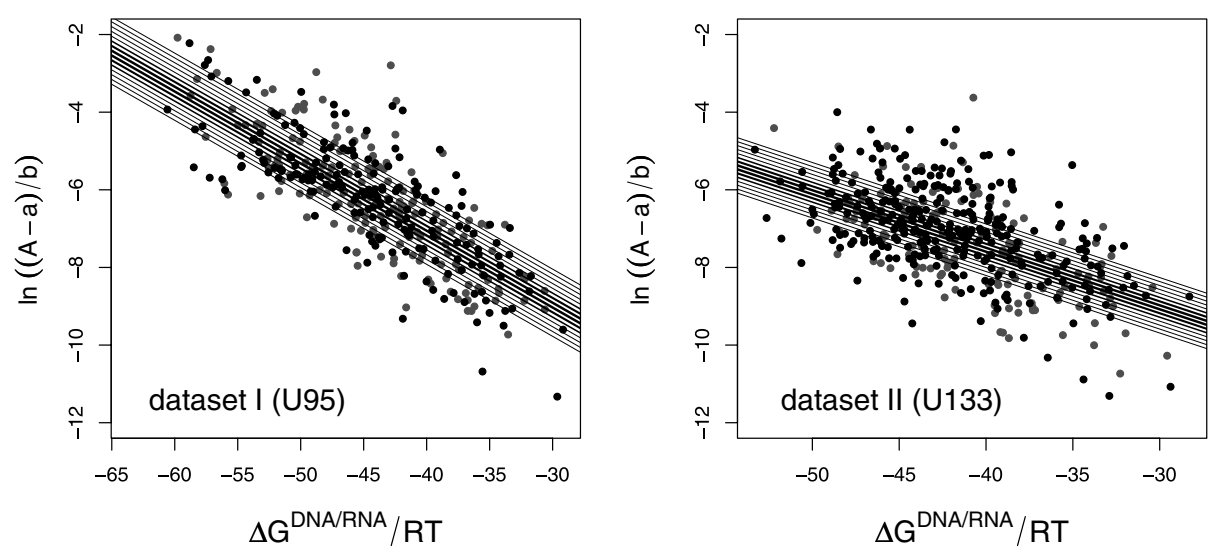

Figure 8. Fits of equation (24) (Model 2) to the parameter $A$ of the hyperbolic response function fits of both PM (black) and MM (red) data for datasets I and II. The parameters $a$ and $b$ are from table 5 . The plotted lines correspond to the range $6 \leqslant n_{\text {pyr }} \leqslant 20$ of pyrimidine counts, with $n_{\text {pyr }}$ increasing from bottom to top.

folding energy of any of the probes, implying that the probefolding effect is effectively linear for dataset I. On the other hand, the fitted value of $\mu_{\alpha}$ in Model 5 for dataset II is -6.14 , which is below the range $-5.49<\Delta g^{\text {DNA-fold }}<3.11$ of calculated probe-folding energies for the U133 microarray, implying that the probe folding is switched off for dataset II. There is no obvious reason why the probe-folding parameter $\mu_{\alpha}$ should shift markedly from one spike-in experiment to another, given that, although the U133 probeset nucleotide sequences are completely redesigned from those of the U95 microarray, the experimental protocol and geometry of the microarray should be similar. One, albeit prosaic, explanation may simply be that dataset II is noisier (see table 1) and the probe-folding effect has been lost in the noise.

Fitted parameter values of Models 2 and 5 are given in table 5. As expected from the above argument, the fitted parameters $c_{1}, c_{2}$ and $c_{3}$ for dataset II differ very little between Models 2 and 5. Fits of Model 2 to the data are shown in figure 8 .

\subsection{The horizontal scale parameter}

The parameter $K$ sets the horizontal scale of the hyperbolic response function (1). $K^{-1}$ is an estimate of the spike-in concentration required to give a fluorescence intensity half way between the background, zero concentration level and the asymptotic, infinite concentration level. Our model in section 3 explains $K$ as an effective rate reaction constant which is determined by the reactions occurring during the hybridization step, and which is unaffected by the washing step. As was the case for the vertical scale parameters, much of the information required to evaluate $K$ from first principles is unknown, and so we try for an ansatz based on probe sequences and free binding energies.

Equations (16), (5) and (9) give $K$ in terms of reaction rate constants and concentrations of reactants. In general, each term $K^{r}$ or $X^{r}$ occurring in equation (16), where $r$ labels one of the reactions in table 3 , is a sum of terms of the form const $\times \mathrm{e}^{-\Delta G_{i}^{r} / R T}$, weighted by the concentration of the reactant $i$. Once again we will be guided by Heim et al's numerical estimate of $X^{\text {bulk }}$ [14], and approximate each sum as a single exponential term. Thus we write

$$
K^{r} \text { or } X^{r}=\exp \left[\lambda_{r}\left(\mu_{r}-\Delta g^{r}\right)\right]
$$

where $\mu_{r}$ and $\lambda_{r}$ are fitting parameters and $\Delta g^{r}=\Delta G^{r} / R T$, with the effective binding energy $\Delta G^{r}$ for each reaction is estimated from some external physical model. With the sign convention that $\Delta G^{r}$ is defined negative for a bound state, each $\lambda_{r}$ is expected to be positive.

First, consider dataset III for which the complex nonspecific background is absent. In equation (16) we can set the non-specific binding terms $X^{\text {bulk }}$ and $X^{\mathrm{NS}}$ to zero, giving

$$
\ln K=\ln K^{\mathrm{S}}-\ln \left(1+K^{\text {Sfold }}\right)-\ln \left(1+K^{\text {Pfold }}\right) .
$$

The rate constants are modelled as

$$
\begin{gathered}
K^{\mathrm{S}}=\exp \left[\lambda_{\mathrm{S}}\left(\mu_{\mathrm{S}}-\Delta g^{\mathrm{DNA} / \mathrm{RNA}}\right)\right], \\
K^{\text {Sfold }}=\exp \left[\lambda_{\text {Sfold }}\left(\mu_{\text {Sfold }}-\Delta g^{\mathrm{RNA} / \mathrm{RNA}}\right)\right], \\
K^{\text {Pfold }}=\exp \left[\lambda_{\text {Pfold }}\left(\mu_{\text {Pfold }}-\Delta g^{\text {DNA-fold }}\right)\right] .
\end{gathered}
$$

For the free binding energy $\Delta G^{\mathrm{DNA} / \mathrm{RNA}}$ we use the nearestneighbour stacking model parameters of Sugimoto et al [22], for $\Delta G^{\mathrm{RNA} / \mathrm{RNA}}$ we use Xia et al's nearest-neighbour stacking parameters for RNA binding to RNA [23] and for $\Delta G^{\text {DNA-fold }}$ we use Zuker's Mfold web server [24]. The Mfold web server also has the facility to calculate folding energies of RNA targets. However, since for RNA target folding we are interested in the propensity for the 25-mer stretch of target complimentary to a given probe to bind with any segment of the much longer target RNA (or possibly another RNA molecule), we believe it is more appropriate to model target folding in the solution using an RNA-to-RNA binding energy.

To try and understand the relative importance of each of the effects contributing to the effective rate constant $K$, we have analysed a set of models containing nested pairs, the relationship between which is illustrated in figure 9:

Model 0: $\ln K=k_{0}+\epsilon$,

Model 1: $\ln K=\lambda_{\mathrm{S}}\left(\mu_{\mathrm{S}}-\Delta g^{\mathrm{DNA} / \mathrm{RNA}}\right)+\epsilon$, 

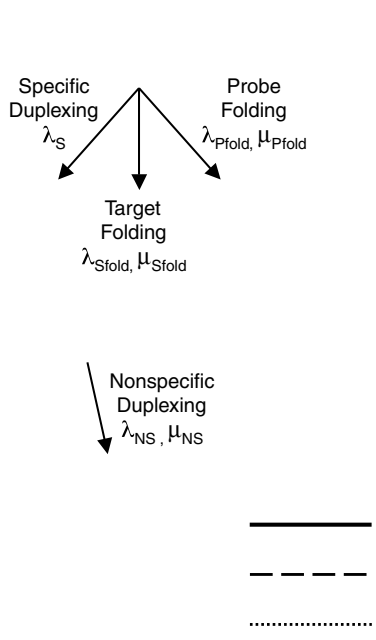

Dataset I

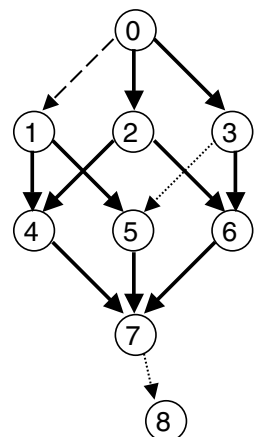

(8)
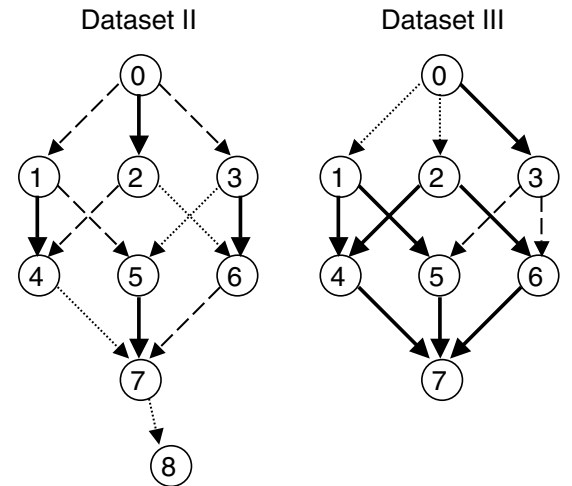

Significant at the $0.01 \%$ level $\left(\mathrm{P}\right.$-value $\left.<10^{-4}\right)$

Significant at the $1 \%$ level $\left(10^{-4}<\mathrm{P}\right.$-value $\left.<10^{-2}\right)$

Not significant at the $1 \%$ level $\left(\mathrm{P}\right.$-value $\left.>10^{-2}\right)$

Figure 9. The relationship between the nine models fitting the effective rate constant $K$, namely equations (36)-(43) and (46). The directions of the arrows indicate the extra effects included in going from a simpler, nested model to a more complicated model, and the style of the arrows indicate $p$-values from table 7. Smaller $p$-values indicate that the extra parameters in the more complicated model are significant. The fitted parameters corresponding to the favoured model, Model 7, are given in table 5.

Model 2: $\ln K=k_{0}$

$$
-\ln \left\{1+\exp \left[\lambda_{\text {Sfold }}\left(\mu_{\text {Sfold }}-\Delta g^{\text {RNA } / \text { RNA }}\right)\right]\right\}+\epsilon,
$$

Model 3: $\ln K=k_{0}$

$$
-\ln \left\{1+\exp \left[\lambda_{\text {Pfold }}\left(\mu_{\text {Pfold }}-\Delta g^{\text {DNA-fold }}\right)\right]\right\}+\epsilon,
$$

Model 4: $\ln K=\lambda_{\mathrm{S}}\left(\mu_{\mathrm{S}}-\Delta g^{\mathrm{DNA} / \mathrm{RNA}}\right)$

$$
-\ln \left\{1+\exp \left[\lambda_{\text {Sfold }}\left(\mu_{\text {Sfold }}-\Delta g^{\mathrm{RNA} / \mathrm{RNA}}\right)\right]\right\}+\epsilon,
$$

Model 5: $\ln K=\lambda_{\mathrm{S}}\left(\mu_{\mathrm{S}}-\Delta g^{\mathrm{DNA} / \mathrm{RNA}}\right)$

$$
-\ln \left\{1+\exp \left[\lambda_{\text {Pfold }}\left(\mu_{\text {Pfold }}-\Delta g^{\text {DNA-fold }}\right)\right]\right\}+\epsilon,
$$

Model 6: $\ln K=k_{0}$

$$
\begin{aligned}
& -\ln \left\{1+\exp \left[\lambda_{\text {Sfold }}\left(\mu_{\text {Sfold }}-\Delta g^{\text {RNA } / \text { RNA }}\right)\right]\right\} \\
& -\ln \left\{1+\exp \left[\lambda_{\text {Pfold }}\left(\mu_{\text {Pfold }}-\Delta g^{\text {DNA-fold }}\right)\right]\right\}+\epsilon,
\end{aligned}
$$

Model 7: $\ln K=\lambda_{\mathrm{S}}\left(\mu_{\mathrm{S}}-\Delta g^{\mathrm{DNA} / \mathrm{RNA}}\right)$

$$
\begin{aligned}
& -\ln \left\{1+\exp \left[\lambda_{\text {Sfold }}\left(\mu_{\text {Sfold }}-\Delta g^{\mathrm{RNA} / \mathrm{RNA}}\right)\right]\right\} \\
& -\ln \left\{1+\exp \left[\lambda_{\text {Pfold }}\left(\mu_{\text {Pfold }}-\Delta g^{\text {DNA-fold }}\right)\right]\right\}+\epsilon,
\end{aligned}
$$

where $\epsilon$ is the residual error, which is assumed Gaussian. The first term in each of the Models 1, 4, 5 and 7 could equally well be written as $k_{0}+k_{1} \Delta g^{\mathrm{DNA} / \mathrm{RNA}}$ to make the nesting explicit, but for convenience we use a parameterization based on equation (33). Models 1-3 include only the effects of specific hybridization, target folding in the bulk solution and probe folding, respectively. Models 4-6 include pairwise effects, and Model 7 includes all the three effects. A recurring theme in these models is the functional form of equation (30). Thus, the target- and probe-folding effects 'switch on' once the binding energy is below (i.e. more tightly binding than) thresholds $\mu_{\text {Sfold }}$ and $\mu_{\text {Pfold }}$, respectively, and have the effect of reducing the effective rate constant $K$.

$p$-Values calculated from the $F$-statistic, equation (29), testing the pairwise comparative significance of these models are given for dataset III in the right-hand column of table 7 (see also figure 9). Results are shown for PM data only as no complete set of stacking model parameters for $\Delta G^{\mathrm{DNA} / \mathrm{RNA}}$ with mismatches is available. Comparisons of Model 0 with Models 1-3 indicate that, taken in isolation, the specific hybridization and bulk target-folding effects appear not to be significant, whereas the probe-folding effect appears to be highly significant. This is also illustrated in figure 10 . However, when taken in combination with probe folding, the analysis shows specific binding and target folding to be significant at the 1\% level (see the comparison Models 3-5 and 3-6 in table 7). Thus we accept Model 7 for dataset III. The fitted parameters are given in the right-hand column of table 5 . Note that each $\lambda_{r}$ is positive as required by the model.

For dataset III, the apparent non-significance of the specific hybridization and bulk target-folding effects in Models 1 and 2 can be explained as follows. In Model 7, the bulk folding is a stronger effect than specific hybridization by a factor of $2\left(\lambda_{\text {sfold }} \approx 2 \lambda_{\mathrm{S}}\right.$ in table 5). Furthermore, from equation (30), the bulk-folding effect is opposite in sign to the specific hybridization effect, and only comes into effect for $\Delta g^{\mathrm{RNA} / \mathrm{RNA}}<\mu_{\text {Sfold }}=-73.7$. Also, it turns out that $\Delta g^{\mathrm{DNA} / \mathrm{RNA}}$ and $\Delta g^{\mathrm{RNA} / \mathrm{RNA}}$ are very highly correlated, with a Pearson correlation coefficient of 0.89 . Examination of the first two plots in figure 10 shows a tendency of the data to increase with $\Delta g$ to start with, while the bulk target folding dominates, and then to decrease once the bulk-folding effect switches off and the specific hybridization effect takes over. Attempting to fit a straight line through data which first increases and then decreases has resulted in the conclusion that the term linear in $\Delta G$ in Model I is not significant. A related statement has been made by Carlon and Heim [10], namely that the effective target concentration needs to be appropriately 'rescaled' for those targets with a high binding affinity in the bulk solution in order to see the expected relationship between $K$ and $\Delta G^{\text {DNA/RNA }}$. 

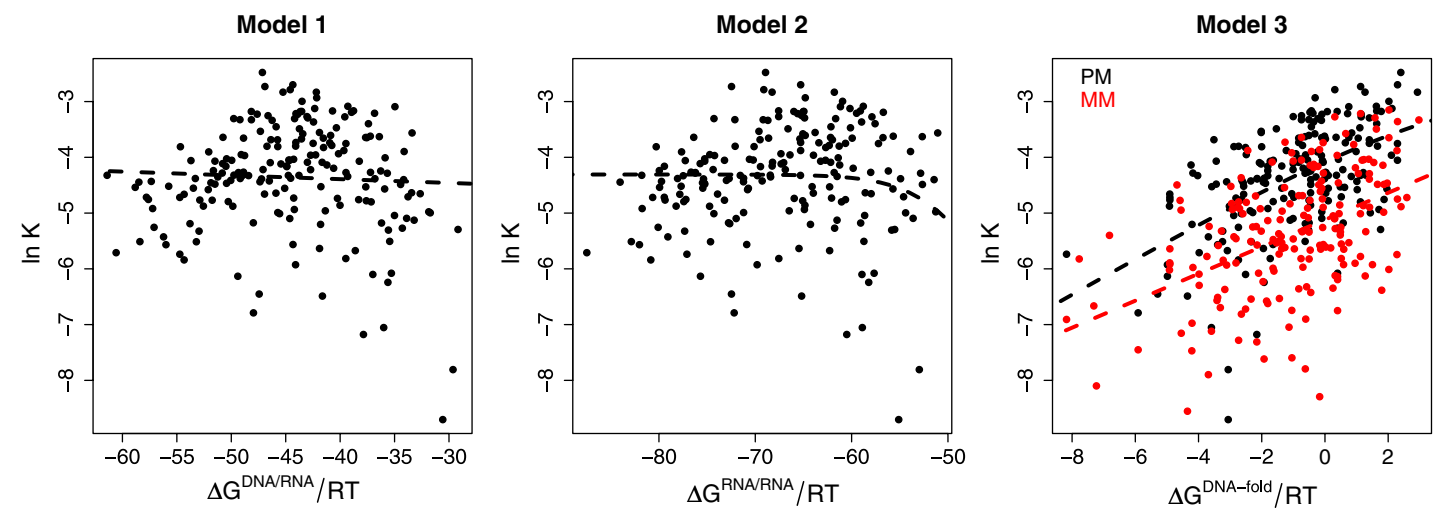

Figure 10. Fits of $\ln K$ estimated from dataset III to Models 1,2 and 3 . Mismatch data are also shown for Model 3 since $\Delta G^{\text {Pfold }}$ can be obtained from the Mfold web site for all probe sequences.

Table 7. $p$-Values testing significance of the extra parameters related to nested pairs of Models $0-8$ fitting the effective rate constant $K$. Smaller $p$-values indicate that the extra parameters in the more complicated model are significant. The number of fitted PM hyperbolic response functions for which all the three parameters $A, B$ and $K$ are positive, and hence the number of points fitted to the models, is 188 for dataset I, 303 for dataset II and 192 for dataset III.

\begin{tabular}{llllc}
\hline & Parameter & Dataset I & Dataset II & Dataset III \\
\hline Model 0 to Model 1: & $\lambda_{\mathrm{S}}$ & 0.00028 & 0.00021 & 0.51 \\
Model 0 to Model 2: & $\lambda_{\text {Sfold }}, \mu_{\text {Sfold }}$ & $3.5 \times 10^{-11}$ & $1.1 \times 10^{-7}$ & 0.32 \\
Model 0 to Model 3: & $\lambda_{\text {Pfold }}, \mu_{\text {Pfold }}$ & $1.6 \times 10^{-12}$ & 0.00011 & $4.8 \times 10^{-15}$ \\
Model 1 to Model 4: & $\lambda_{\text {Sfold }}, \mu_{\text {Sfold }}$ & $<2 \times 10^{-16}$ & $2.3 \times 10^{-6}$ & $1.8 \times 10^{-9}$ \\
Model 1 to Model 5: & $\lambda_{\text {Pfold }}, \mu_{\text {Pfold }}$ & $3.0 \times 10^{-10}$ & 0.0077 & $<2 \times 10^{-16}$ \\
Model 2 to Model 4: & $\lambda_{\mathrm{S}}$ & $4.4 \times 10^{-12}$ & 0.0055 & $5.9 \times 10^{-10}$ \\
Model 2 to Model 6: & $\lambda_{\text {Pfold }}, \mu_{\text {Pfold }}$ & $6.8 \times 10^{-7}$ & 0.089 & $<2 \times 10^{-16}$ \\
Model 3 to Model 5: & $\lambda_{\mathrm{S}}$ & 0.087 & 0.023 & 0.00016 \\
Model 3 to Model 6: & $\lambda_{\text {Sfold }}, \mu_{\text {Sfold }}$ & $1.4 \times 10^{-5}$ & $9.6 \times 10^{-5}$ & 0.00012 \\
Model 4 to Model 7: & $\lambda_{\text {Pfold }}, \mu_{\text {Pfold }}$ & $9.1 \times 10^{-5}$ & 0.056 & $7.7 \times 10^{-14}$ \\
Model 5 to Model 7: & $\lambda_{\text {Sfold }}, \mu_{\text {Sfold }}$ & $3.8 \times 10^{-13}$ & $1.7 \times 10^{-5}$ & $2.0 \times 10^{-5}$ \\
Model 6 to Model 7: & $\lambda_{\mathrm{S}}$ & $7.9 \times 10^{-10}$ & 0.0034 & $2.4 \times 10^{-5}$ \\
Model 7 to Model 8: & $\lambda_{\text {NS }}, \mu_{\text {NS }}$ & 0.016 & 0.23 & \\
\hline
\end{tabular}

We now turn to datasets I and II. In the presence of a complex non-specific background, $X^{\text {bulk }}$ and $X^{\mathrm{NS}}$ are reinstated in equation (16). The bulk hybridization effect will be a sum of exponentials of $\Delta G_{i}^{\mathrm{RNA} / \mathrm{RNA}}$, and its modelling can be absorbed into that for bulk target folding, while the nonspecific effect will be a sum of exponentials of $\Delta G_{i}^{\text {DNA/RNA }}$. Thus we set

$$
\begin{gathered}
K^{\text {Sfold }}+X^{\text {bulk }}=\exp \left[\lambda_{\text {Sfold }}\left(\mu_{\text {Sfold }}-\Delta g^{\mathrm{RNA} / \mathrm{RNA}}\right)\right], \\
X^{\mathrm{NS}}=\exp \left[\lambda_{\mathrm{NS}}\left(\mu_{\mathrm{NS}}-\Delta g^{\mathrm{DNA} / \mathrm{RNA}}\right)\right],
\end{gathered}
$$

which suggests one further model:

Model 8: $\ln K=\lambda_{\mathrm{S}}\left(\mu_{\mathrm{S}}-\Delta g^{\mathrm{DNA} / \mathrm{RNA}}\right)$

$$
\begin{aligned}
& -\ln \left\{1+\exp \left[\lambda_{\text {Sfold }}\left(\mu_{\text {Sfold }}-\Delta g^{\mathrm{RNA} / \mathrm{RNA}}\right)\right]\right\} \\
& -\ln \left\{1+\exp \left[\lambda_{\text {Pfold }}\left(\mu_{\text {Pfold }}-\Delta g^{\text {DNA-fold }}\right)\right]\right. \\
& \left.+\exp \left[\lambda_{\mathrm{NS}}\left(\mu_{\mathrm{NS}}-\Delta g^{\mathrm{DNA} / \mathrm{RNA}}\right)\right]\right\}+\epsilon .
\end{aligned}
$$

Turning to table 7, columns I and II, we discover that the extra parameters introduced to account for non-specific probe-target binding are not significant at the $1 \%$ level (see also figure 9). This surprising result can be explained by the fact that the fitted values of $\mu_{\mathrm{NS}}$ are in both cases close to the maximum value of $\Delta g^{\text {DNA/RNA }}$ within the dataset, so most of the fitted points fall into the $\Delta g^{\mathrm{DNA} / \mathrm{RNA}} \ll \mu_{\mathrm{NS}}$ regime of equation (30), and the effect is adequately covered by the $\lambda_{\mathrm{S}}$ term of Model 7 . To further illustrate the point, fits to Models 1, 2 and 3 are plotted in figure 11. If Model 1 is taken in isolation, $\lambda_{S}$ appears to have the 'wrong' sign, as the non-specific probe-target binding and target folding and binding in solution all combine to dominate the specific binding effect. A similar result is observed for dataset II.

The generally small $p$-values in the first column of table 7 indicate that Model 7 is an appropriate description of the parameter $K$ for dataset I. For dataset II the picture is less clear. In agreement with the analysis of the parameter $\alpha$, the probe folding is in general less significant. Nevertheless, for consistency we list the fitting parameters of Model 7 to both datasets in table 5, while acknowledging there is redundancy in the dataset II parameters.

\subsection{How close are the fits?}

Figure 12 gives some idea of how much information has been lost in the above fits. The plot compares the estimated 

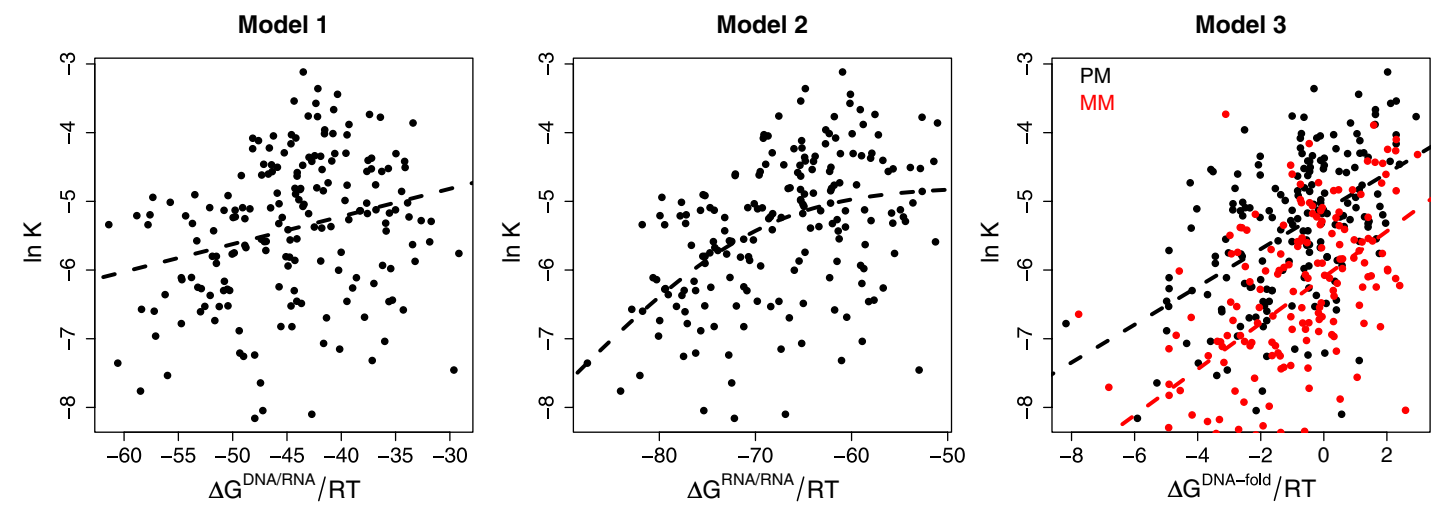

Figure 11. The same as figure 10 for dataset I.
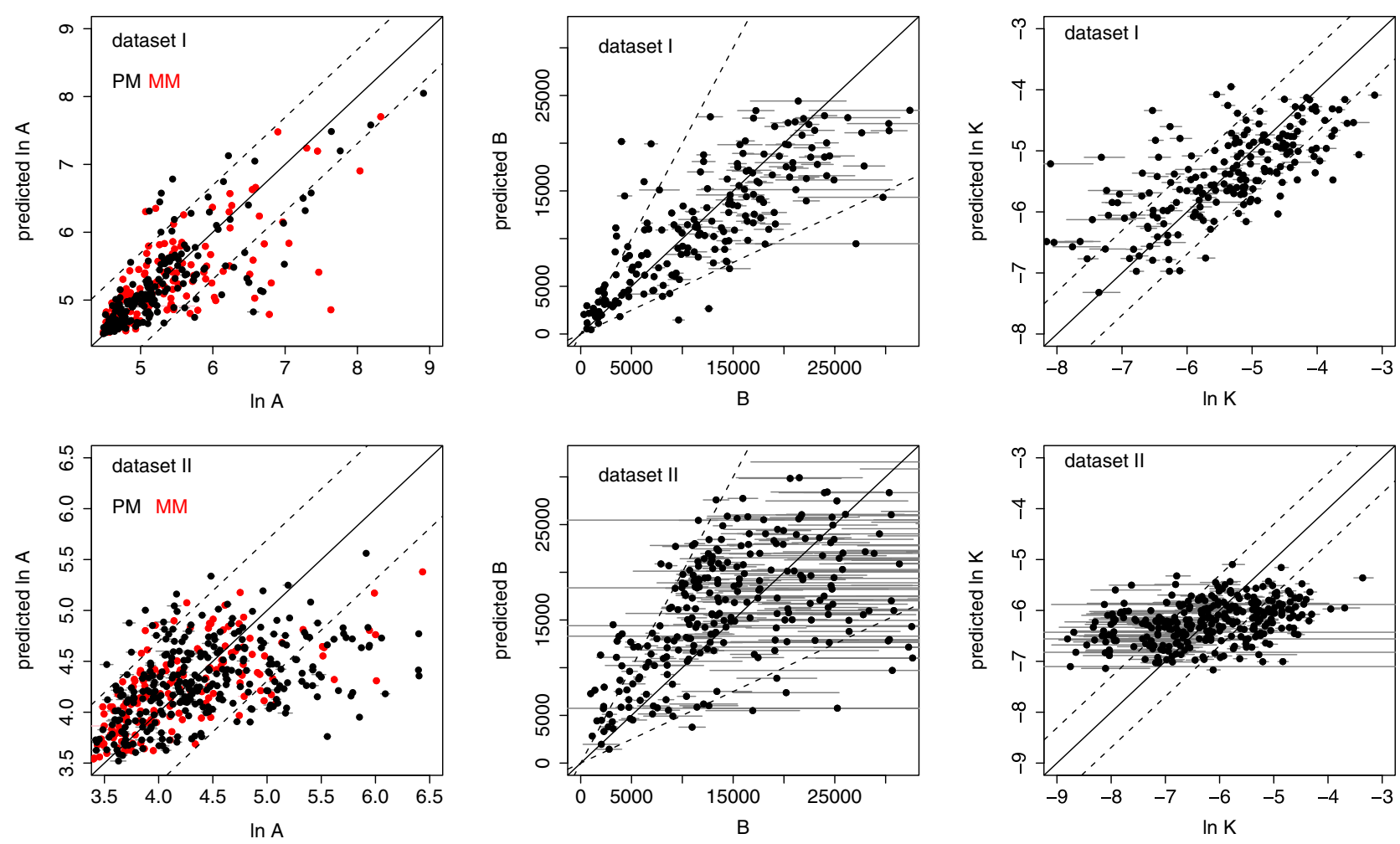

Figure 12. Estimated parameters obtained by fitting the hyperbolic response function (1) to datasets I and II (horizontal axes, together with error bars showing standard errors) plotted against with the values that would be predicted by the quantitative fits of section 5 (vertical axes). The dotted lines indicate a factor of 2 on either side of the diagonal.

parameters $A, B$ and $K$ of the fitted hyperbolic response curves, such as those in figure 1 , with those which would be predicted by the fitting constants and parameters listed in table 5, namely,

$$
\begin{aligned}
& A=a+b \mathrm{e}^{c_{1}+c_{2} \Delta g^{\mathrm{DNA} / \mathrm{RNA}}+c_{3} n_{\mathrm{pyr}}-\ln \left\{1+\exp \left[\lambda_{\alpha}\left(\mu_{\alpha}-\Delta g^{\mathrm{DNA}-\text { fold }}\right)\right]\right\}} \\
& B=a+b \mathrm{e}^{-c_{0} \exp \left(\lambda_{0} \Delta g^{\mathrm{RNA} / \mathrm{RNA}}\right)}-A \\
& K=\frac{\mathrm{e}^{\lambda_{\mathrm{S}}\left(\mu_{\mathrm{S}}-\Delta g^{\mathrm{DNA} / \mathrm{RNA}}\right)}}{\left[1+\mathrm{e}^{\lambda_{\text {Sfold }}\left(\mu_{\text {Sfold }}-\Delta g^{\mathrm{RNA} / \mathrm{RNA}}\right)}\right]\left[1+\mathrm{e}^{\lambda_{\mathrm{Pfold}}\left(\mu_{\text {Pfold }}-\Delta g^{\mathrm{DNA}-\text { fold }}\right)} .\right.} .
\end{aligned}
$$

Dotted lines on either side of the diagonal are the boundary of the region within which predicted values do not differ from the original fitted parameters by more than a factor of 2 . A clear majority of estimates of $A$ and $B$ fall within this range. Clearly the most difficult parameter to explain adequately is the horizontal scale $K$, owing to the large number of contributing chemical reactions. In general, dataset II has proved to be more problematic than dataset I, probably because the concentration range tested does not extend far enough into the saturation regime to demonstrate a clear hyperbolic isotherm. 

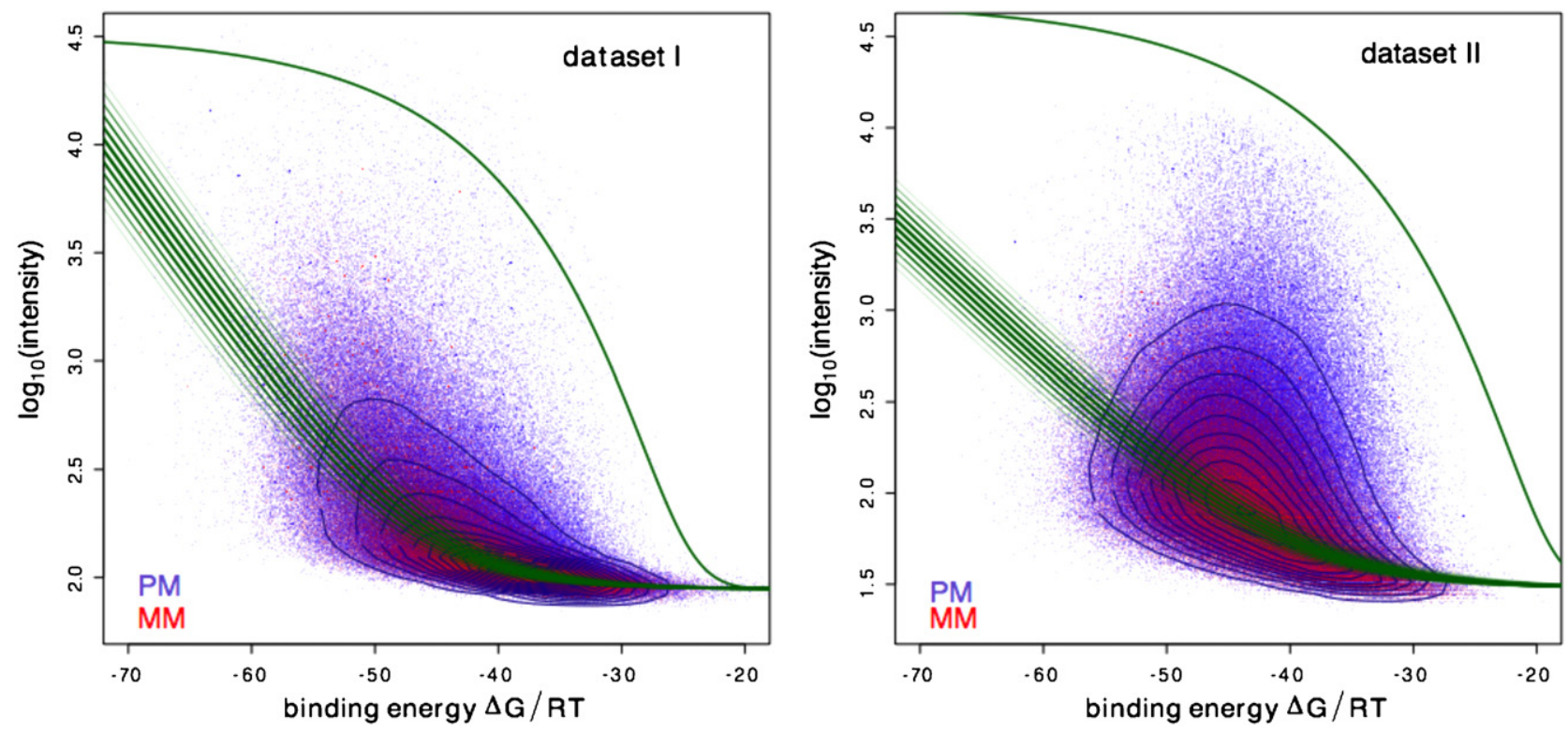

Figure 13. Scatter plots of measured fluorescence intensities against the theoretical DNA/RNA free binding energies. The upper curve is the fit to $A+B$ of equation (48), and the lower set of curves are the fits to $A$ of equation (47) for pyrimidine counts $6<n_{\text {pyr }}<20$, with $n_{\text {pyr }}$ increasing from bottom to top. Also shown are contour lines of the density of points.

\section{Parameter prediction}

For the above model to be of value in constructing a practical algorithm for inferring target concentrations, some or all of its parameters should ideally be predictable using only the information available to experimental biologists. That available information consists of fluorescence intensities for the complete set of features on each microarray used in an experiment, the probe sequences of each feature, and any parameters associated with the experimental protocol. In contrast, the fitted parameters of table 5 were obtained from spike-in experiments. Comparing datasets I and III in figures 4 and 5, one sees for instance that the unknown nature of the complex background has a profound effect on the parameters $A$ and $K$ of the hyperbolic response function. At first sight it appears one may need a new set of spike-in data for each experiment, which is clearly not a practical consideration. However, we argue here that if one exploits the distribution of fluorescence intensities from the entire microarray, an estimation of the vertical scale parameters at least may be possible.

In the following qualitative description, we propose a two-step process for the vertical scale parameters, in which the physical background $a$ and the maximum intensity $b$ for a microarray are first determined from the entire distribution of intensities over the microarray. The intensities $I(x)$ can then be scaled to the dimensionless coverage fraction $\theta(x)$ via equation (2), and one is left with the remaining problem of estimating the parameters $\alpha$ and $\beta$, which are driven by the chemical reactions of table 3 .

To estimate $a$ and $b$, consider the histograms in figure 6 . For both datasets I and II, our estimate of the physical background $a$, based on hyperbolic response curves derived from spike-in data, is close to two standard deviations above the minimum measured fluorescence intensity. Assuming an experiment consisting of a number of technical replicates of each hybridization setup, the data can be quantile normalized across replicates. A representative minimum intensity $a_{\min }$ can be obtained by fitting a suitable smooth curve to the logged histogram (i.e. the lower panel of figure 6), and the coefficient of variation in the data $\eta$ easily estimated from the replicate intensity values over the whole microarray. $(1+2 \eta) a_{\min }$ then gives an estimate of $a$.

Estimating $b$ from the histogram proves to be quite difficult because of the gradual tail at its right-hand end. With some experimentation we find that a cubic fit to the logged histogram over the range $[l+0.25(u-l), l+0.875(u-l)]$, where $l$ and $u$ are the lower and upper extremities of the histogram, crosses the $\log _{10}$ (count) $=0$ line close to two standard deviations above the previously obtained estimate of $a+b$. Calling this point $(a+b)_{\max }$, an estimate of $a+b$ is then $(1-2 \eta)(a+b)_{\max }$. However, we find that such a method is highly sensitive to the range over which the cubic is fitted.

To gain some insight into how $\alpha$ and $\beta$ may be estimated, consider the scatter plot, figure 13, of fluorescence intensities against the theoretical free binding energy $\Delta G^{\mathrm{DNA} / \mathrm{RNA}}$ obtained from the probe letter sequences using the nearestneighbour stacking model [22]. Superimposed on these plots are the fits from equations (47) and (48) to the asymptotic saturation intensity $A+B$ and background intensity at zero spike-in concentration $A$, using the parameters of table 5 . According to our model, the asymptote curve should form an upper envelope to the data, with some slight leakage across the envelope due to the finite coefficient of variation in the data. Because the vast majority of the genes are not expressed in RNA samples taken from a typical cell, most of the data are expected to lie along, or close to, the lower set of background intensity curves. Indeed this is precisely what 
is seen. Conversely, in the absence of spike-in data, there is potential to estimate the upper, asymptote intensity curve by fitting an envelope to the data and the lower, background intensity curve by fitting a curve through the ridge of the scatter plot's contour lines. In principle, these fitted curves, together with $a$ and $b$ then determine the estimates of $\alpha$ and $\beta$ for each feature on the microarray.

\section{Conclusions and outlook}

This paper concentrates mainly on the immediate aim of understanding the physical processes at work in the operation of microarrays, but in doing so highlights some of the challenges and, we hope, gives some guidance, for meeting the ultimate aim of providing an algorithm for converting the set raw fluorescence intensities from microarrays to absolute target concentrations.

The model we have examined includes the effects of specific and non-specific hybridization, folding and hybridization in the bulk solution of target RNA, the folding of probes at the microarray surface, and the removal of signal during the post-hybridization step. It leads to the hyperbolic response curve (or the Langmuir isotherm) of equation (1) with three fitting parameters $A, B$ and $K$, which depend on a set underlying physical parameters including chemical reaction rate constants, washing survival functions and RNA target concentrations. In more practical terms, all the three parameters will depend on the probe letter sequence, whether the probe is PM or MM, the nature of the complex non-specific background, and experimental protocols such as hybridization temperature and washing times. Determining the parameters only from information likely to be known to biologists in a practical situation, as opposed to a highly controlled spike-in experiment, remains a formidable task.

The model is tested against the Affymetrix U95 spike-in datasets with and without a complex non-specific background and the Affymetrix U133 spike-in dataset. In general, an agreement with a hyperbolic response curve is excellent for the U95 spike-in datasets and reasonable for the U133 spikein dataset (see table 1).

The response function parameters $A$ and $B$ set the vertical scale of the isotherm, that is, the scale of the measured fluorescence intensities. The parameter $A$ is a combination of a relatively straightforward physical background, and a nontrivial contribution from the complex non-specific background. It is important to understand the nature of the non-specific background component as it is responsible for the 'bright mismatches' problem which complicates the naive PM-MM subtraction scheme used in the MAS5 algorithm, for instance, for dealing with non-specific hybridization. Our analysis shows that the DNA/RNA binding energy, pyrimidine content, and (in the case of U95 dataset) the folding of probes, all contribute significantly to the value of this parameter. The dependence of $A$ on binding energies and pyrimidine count is illustrated in figure 8 .

The parameter $B$ (or, more precisely, the combination $A+B$, where $B \gg A$ in general) is mainly concerned with the asymptote at high spike-in concentration, which, according to our model, is driven by the washing step. The qualitative prediction that it should be less for a mismatch feature than for a perfect-match feature in a PM/MM pair is verified for all the three datasets in figure 3. Its quantitative behaviour as a function of specific probe-target binding energies, using bulk solution free binding energies as a guide, is verified in figure 7.

The parameter $K$ can be thought of as an effective overall reaction rate. It sets the horizontal scale of the isotherm, that is, the scale of the specific target concentration. If an algorithm to determine absolute target concentrations is to be constructed, it is necessary to understand this parameter. Because of the large number of hybridization reactions which have the potential to contribute it is by far the hardest of the three parameters to explain effectively. The model predicts that it is affected by all of the reactions listed in table 3 occurring in the bulk hybridization solution and at the microarray surface, but is unaffected by the dissociation during the washing phase. Analysis to determine which reactions are significant is complicated by the fact that the effect on $K$ of non-specific hybridization and probe and target folding act in the opposite direction to that of specific binding, so obscuring the effects. Nevertheless, our analysis indicates that all of the hybridization reactions considered are potentially significant contributors.

A common practice in previous studies [7, 10, 14-17] has been to invert the fits of hyperbolic response curves to recover spike-in target concentrations in order to test the predictive ability of models. We have deliberately refrained from doing so here, as one of the results of this study has been to demonstrate the strong dependence of the parameters of the isotherm on the (in practice unknown) complex non-specific background. Recovering spike-in concentrations using fitting parameters which implicitly contain information about the background belonging to a particular dataset is an inherently circular argument and is guaranteed to give unrealistically good results.

Instead, in section 6, we address the problem of determining the hyperbolic response function parameters from information likely to be available to biologists in a typical microarray experiment, that is, fluorescence intensities for the complete set of features on each microarray, the probe sequences, and parameters associated with the experimental protocol. We argue that information for the vertical scale parameters is in principal implicitly contained in the distribution of intensities across the microarray by partitioning the intensities by quantities which can be estimated from probe sequences such as probe-target binding energies, probe folding energies and probe pyrimidine content. Determination of the horizontal scale parameter is a more formidable and open problem.

Ultimately, our aim is to find practical algorithms for biological analysis through an improved understanding of the physics of microarrays. A problem encountered in this paper, particularly with analysis of the horizontal scale parameter of the isotherm $K$, has been the difficulty encountered unravelling mutual correlations and compensations between competing effects. In such cases one can never be totally certain, given 
the available data, that the physical interpretation is correct. However, one could argue that it is not necessary, nor possibly even helpful, to know all of the contributing physical effects in order to meet our ultimate aim. If, for instance, our interpretation of the significance analysis of various models of the parameter $K$ in section 5.2 is correct, we discover that we often do just as well by modelling a number of physical effects by a single effective term. In general, the lesson is that it is important to strike a balance between attempting to understand and incorporate all physical aspects on the one hand, and relying on empirically determined effective models on the other.

The above analysis has concentrated on Affymetrix gene expression microarrays. Genomic microarrays come in a variety of types for a variety of tasks. As well as gene expression arrays there are tiling arrays, which interrogate large contiguous tracts of genome rather than specific genes, resequencing arrays designed to detect the location of mutations and single nucleotide polymorphisms (SNPs), SNP arrays which are used for genotyping, and non-DNA arrays such as protein arrays designed to identify protein-protein interactions and antibody arrays for detecting antigens. In addition to this there exist a number of technologies including photolithographic deposition, inkjet printing and fabrication of probes onto micro-beads.

In each case they share the common property that they detect large biological molecules of specific known letter sequences via their binding to complementary sequences attached to a solid surface. In each case they will almost invariably share the common problems of the non-specific hybridization, saturation and bulk solution target hybridization and folding dealt within this work. The physico-chemical model we have explored is consistent with spike-in data over a broad range of target concentrations and should serve as a starting point for a variety of microarray types and platforms.

\section{Acknowledgments}

The author thanks Hans Binder, Susan Wilson and Yvonne Pittelkow for useful discussions and advice.

\section{Glossary}

Hybridization. The reversible chemical reaction by which target molecules in the solution bind to probes attached to the microarray surface to form duplexes.

Microarray. A high-throughput device for detecting the presence of large biological molecules (DNA, RNA or proteins) of specific known letter sequences via their binding to molecules of complementary sequences attached to a solid surface. They are high-throughput in the sense that large numbers of sequences are tested for in a single device. The microarrays discussed here are oligonucleotide gene expression microarrays, that is, they have short DNA probes and are intended for the detection of expressed genes through their messenger RNA.
Non-specific hybridization. The hybridization of target molecules with sequences other than those of the intended sequence. Sometimes 'non-specific' is used to mean 'non-PM-specific', that is, hybridization of target molecules which are not complementary to the PM sequence, irrespective of whether they are binding to the PM or MM member of a probe pair. PM and MM are defined in section 2.

Probe. A biological molecule attached to the microarray surface during fabrication.

Spike-in experiment. An experiment in which known concentrations of a specific set of target molecules are artificially added to a solution not otherwise containing those specific targets, and the solution hybridized onto microarrays.

Target. A biological molecule in the solution hybridized onto the microarray during a laboratory experiment.

\section{References}

[1] http://www.affymetrix.com/support/technical/ sample_data/datasets.affx

[2] Binder H 2006 Thermodynamics of competitive surface adsorption on DNA microarrays J. Phys.: Condens. Matter 18 S491-523

[3] Binder H, Kirsten T, Hofacker I L, Stadler P F and Loeffler M 2004 Interactions in oligonucleotide hybrid duplexes on microarrays J. Phys. Chem. 108 18015-25

[4] Binder H, Kirsten T, Loeffler M and Stadler P F 2004 Sensitivity of microarray oligonucleotide probes: variability and effect of base composition J. Phys. Chem. 108 18003-14

[5] Binder H and Preibisch S 2005 Specific and nonspecific hybridization of oligonucleotide probes on microarrays Biophys. J. 89 337-52

[6] Binder H and Preibisch S 2006 Genechip microarrays-signal intensities, RNA concentrations and probe intensities J. Phys.: Condens. Matter 18 S537-66

[7] Burden C J, Pittelkow Y E and Wilson S R 2004 Statistical analysis of adsorption models for oligonucleotide microarrays Stat. Appl. Genetics Mol. Biol. 3 (article 35)

[8] Burden C J, Pittelkow Y E and Wilson S R 2006 Adsorption models of hybridisation and post-hybridisation behaviour on oligonucleotide microarrays J. Phys.: Condens. Matter 18 5545-65

[9] Burden C J, Pittelkow Y E and Wilson S R 2007 Statistical analysis and physical modelling of oligonucleotide microarrays Math. Mod. Biol. Syst. vol I ed I A Deutsch, L Brusch, H Byrne, G de Vries and H-P Herzel (Boston, MA: Birkhäuser) pp 333-46

[10] Carlon E and Heim T 2006 Thermodynamics of RNA/DNA hybridization in high-density oligonucleotide microarrays Physica A 362 433-49

[11] Carlon E, Heim T, Wolterink J K and Barkema G T 2006 Comment on 'Solving the riddle of the bright mismatches: labelling and effective binding in oligonucleotide arrays' Phys. Rev. E 73063901

[12] Forman J E, Walton I D, Stern D, Rava R P and Trulson M O 1998 Thermodynamics of duplex formation and mismatch discrimination on photolithographically synthesised oligonucleotide arrays Molecular Modeling of Nucleic Acids (ACS Symposium Series vol 682) ed N B Leontis and J SantaLucia (Washington, DC: American Chemical Society) pp 206-28 
[13] Halperin A, Buhot A and Zhulina E B 2004 Sensitivity, specificity and the hybridization isotherms of DNA chips Biophys. J. 86 718-30

[14] Heim T, Tranchevent L-C, Carlon E and Barkema G T 2006 Physical-chemistry-based analysis of Affymetrix microarray data J. Phys. Chem. B 11s0 22786-95

[15] Hekstra D, Taussig A R, Magnasco M and Naef F 2003 Absolute mRNA concentrations from sequence-specific calibration of oligonucleotide arrays $\mathrm{Nucl}$. Acids Res. 31 1962-8

[16] Held G A, Grinstein G and Tu Y 2003 Modeling of DNA microarray data by using physical properties of hybridization Proc. Natl Acad. Sci. 100 7575-80

[17] Held G A, Grinstein G and Tu Y 2006 Relationship between gene expression and observed intensities in DNA microarrays-a model study Nucl. Acids Res. 34 e70

[18] Irizarry R A et al 2003 Exploration, normalization and summaries of high density oligonucleotide array probe level data Biostatistics 4 249-64

[19] Matveeva O V, Shabalina S A, Nemtsov V A, Tsodikov A D, Gesteland R F and Atkins J F 2003 Thermodynamic calculations and statistical correlations for oligo-probe design Nucl. Acids Res. 31 4211-7

[20] Naef F and Magnasco M O 2003 Solving the riddle of the bright mismatches: labelling and effective binding in oligonucleotide arrays Phys. Rev. E 68011906

[21] Skvortsov D, Abdueva D, Curtis C, Schaub B and Tavaré S 2007 Explaining differences in saturation levels for Affymetrix Genechip ${ }^{\mathbb{B}}$ arrays Nucl. Acids Res. 35 4154-63

[22] Sugimoto N, Nakano S, Katoh M, Matsumura A, Nakamuta H and Ohmichi T 1995 Thermodynamic parameters to predict stability of RNA/DNA hybrid duplexes Biochemistry 34 11211-6

[23] Xia T, SantaLucia J, Burkard M E, Kierzek R, Schroeder S J, Jiao X, Cox C and Turner D H 1998 Thermodynamic parameters for an expanded nearest-neighbor model for formation of RNA duplexes with Watson-Crick base pairs Biochem. 37 14719-35

[24] Zuker M 2003 Mfold web server for nucleic acid folding and hybridization prediction Nucl. Acids Res. 31 3406-15 http://frontend.bioinfo.rpi.edu/applications/mfold/ 